\title{
Detecting Link Failures in Complex Network Processes Using Remote Monitoring
}

\author{
R.Dhal ${ }^{\mathrm{a}, 1, *}$, J. A. Torres ${ }^{\mathrm{b}, 1}$, S.Roy $^{\mathrm{c}, 1, * *}$ \\ ${ }^{a}$ EPIS Inc, $1353572^{\text {nd }}$ Ave., Suite 165, Tigard, OR 97223 \\ ${ }^{b}$ Ladrón de Guevara E11253, PO. Box 17-01-2759, Quito, Ecuador \\ ${ }^{c}$ EME 402, PO BOX 642752, Washington State University, Pullman, WA, 99164-2752
}

\begin{abstract}
We study whether local structural changes in a complex network can be distinguished from passive remote time-course measurements of the network's dynamics. Specifically the detection of link failures in a network synchronization process from noisy measurements at a single network component is considered. By phrasing the detection task as a Maximum $A$ Posteriori Probability hypothesis testing problem, we are able to obtain conditions under which the detection is 1) improved over the a priori and 2) asymptotically perfect, in terms of the network spectrum and graph. We find that, in the case where the detector has knowledge of the network's state, perfect detection is possible under general connectivity conditions regardless of the measurement location. When the detector does not have state knowledge, a remote signature permits improved but not perfect detection, under the same connectivity conditions. At its essence, detectability is achieved because of the close connection between a network's topology, its eigenvalues and local response characteristics.
\end{abstract}

Keywords: Estimation in Networks; Decision/Estimation Theory; Fault Detection/Diagnosis; Synchronization.

\section{Introduction}

Network synchronization models - which describe coordination or equalization of network components' states via local interactions - are descriptive of both interesting physicalworld processes and distributed consensus/agreement algorithms [1, 2, 3, 4]. These synchronization models have proved influential in several disciplines, including in characterizing emergent behaviors in nature [5], analyzing traditional engineered networks (e.g., power, transportation and Internet $[6,7]$ ), and designing algorithms for distributed cyber-systems such as wireless sensor networks [8].

\footnotetext{
${ }^{*}$ Corresponding author

** Principal corresponding author

Email addresses: rahuldhal@epis.com (R.Dhal), jabadtor@eecs.wsu.edu (J. A. Torres), sroy@eecs.wsu.edu (S.Roy)

${ }^{1}$ This work was partially supported by NSF Grants ECS-0901137,CNS-1035369, and CNS-1058124.

Preprint submitted to Physica A: Statistical Mechanics and its Applications

March 28, 2015
} 
Many physical-world and cyber-network synchronization processes operate in complex environments, which can effect local topological changes. These local changes can induce network-wide propagative impacts that modify the network's core function, and hence there is a motivation for quickly detecting topological changes. Here, we study whether local changes in a synchronization network's graph can be detected using only passive monitoring at a few network locations.

This study contributes to a growing research effort on inferring complex network topologies (and/or topological changes), either from partial knowledge of the topology or by leveraging a dynamics imposed on the network. One approach taken in the literature is to impose a dedicated algorithm for identifying particular topological changes, e.g. cuts or local damage $[9,10,11]$. Entirely structure-based approaches have also been widely studied, such as data mining techniques to reconstruct a full network topology from partial knowledge [12]. A third approach, which is more closely connected to our focus here, is to use passive measurements of the network's dynamics to infer modal or topological characteristics $[13,14,15,16,17,18]$. These techniques fundamentally leverage the network's native dynamical responses for inference. While our study is aligned with this perspective, we are particularly concerned with detecting local structural changes (specifically link failures) using measurements taken only at a very few network locations, which requires us to exploit remote signatures. In particular, this study examines whether a monitor can infer link failures in a discrete-time network synchronization process from local noisy temporal response data. This type of problem arises e.g. in infrastructure-control and security applications, where stakeholders may only be able to probe the network at a few points, and depend on the propagative impact of topological changes for detection.

The approach taken here is to phrase the problem of inferring link failures as a Maximum A Posteriori Probability (MAP) detection problem, in which the monitor seeks to distinguish between alternative failure hypotheses using its noisy local response data, with or without knowledge of the network's dynamical state. The key contribution of the work is to characterize the performance of the MAP detector in terms of the graph topology of the synchronization process, and the locations of the failure and measurement relative to the graph. Our analysis shows that propagative responses caused by local structural changes allow the monitor to distinguish failures from remote measurement signatures, under broad network-connectivity conditions. In the case where the observer has knowledge of the state, perfect detection is possible asymptotically as long as the network is strongly connected. In the unknown-state case, perfect inference is typically not possible, but the measurement data permits effective inference as long as the measurement noise is limited.

Our analysis also shows that detectability, and the time required for detection, fundamentally depends on how the network spectrum changes due to the failure of links. These dependencies are related to, and informed by, the wide literature connecting a network's spectrum with its graph structure (e.g. [19]). Of particular note, the detection task can be viewed as an extension of the inverse problem phrased as Can one hear the shape of a network?, which asks if the graph eigenvalue uniquely identifies the underlying topology [20]. Here we take the further step of using sparse local measurements of a network's dynamics to identify a change in the topology, which is seen to be possible precisely if the spectrum changes. The spectral analysis of detectability also admits an interesting interpretation from the perspective of [21], which shows that networks with networks with the 
same Laplacian eigenvalues can have substantially different synchronization behavior.

The rest of the article is organized as follows. In Section 2 we introduce the linkfailure detection problem, the MAP detection methodology, and metrics for quantifying detection performance. We differentiate our analysis on the MAP scheme into two cases based on the monitor's knowledge of the initial state. In Section 3 the performance of the MAP scheme is analyzed when the initial state is exactly known. Section 4 focuses on the case where the initial state is unknown and modeled a random variable. Finally, illustrative examples are given in Section 5. Our main focus throughout is to develop graph-theoretic insights into the performance of the MAP detector.

\section{Problem Description}

In this section, we describe the network-synchronization model, pose the problem of detecting (inferring) link failures, and introduce the MAP detection approach.

\subsection{Nominal Networked Synchronization Process}

Nominally, we consider a synchronization dynamics defined on a weighted, directed graph (or digraph) $\Gamma=(\mathcal{V}, E: W)$. Here, $\mathcal{V}$ is a set containing the $n$ vertices in the graph, which we label $1,2, \cdots, n$. $E$ is a set of directed edges or arcs, each of which is an ordered pair of distinct vertices (say $(i, j)$, for the edge from vertex $i$ to vertex $j$ ). Each edge $(i, j)$ in the graph has associated with it a positive weight $w_{i j}$, as specified in the weight set $W$. We concisely represent an arc (directed edge) and its associated weight as $\left(i, j: w_{i j}\right)$. We refer to $i$ and $j$ as the starting and trailing end of the directed edge $(i, j)$.

A nominal synchronization process is defined on $\Gamma$. Specifically, a process with $n$ components is considered, where component $i$ corresponds to vertex $i$ in $\Gamma$. Edges in $\Gamma$ correspond to links in the network synchronization process. Each network component $k$ has associated with it a synchronization state $x_{k}[t]$, which evolves along a discrete time axis $t \in Z^{+}$. At each time step, component $k$ 's state evolves based on a weighted average of differences from other components' states, where the weights are specified by the directed edges into vertex $k$ in $\Gamma$. Specifically, $x_{k}[t]$ is nominally governed by:

$$
x_{k}[t+1]=x_{k}[t]+\sum_{i \in \mathbb{N}_{k}} w_{i k}\left(x_{i}[t]-x_{k}[t]\right)
$$

where the neighborhood set $\mathbb{N}_{k}$ contains the indices of all vertices $i$ that are adjacent to $k$ in the graph $\Gamma$ (i.e., such that $(i, k)$ is an edge). We say that there is a link from component $i$ to component $k$ in the network if $w_{i k}>0$. Conceptually, the update (1) serves to correct differences between the components' local states, and hence can achieve synchronization upon repeated application.

The updates for the $n$ components can be assembled into the following vector difference equation:

$$
x[t+1]=A_{0} x[t]
$$

where $x[t]=\left[\begin{array}{llll}x_{1}[t] & x_{2}[t] & \cdots & x_{n}[t]\end{array}\right]^{\prime}$, the notation $(\cdot)^{\prime}$ indicates a transpose, the state matrix $A$ can be written as $A_{0}=I-L, I$ is an identity matrix, and $L$ is the weighted in-degree Laplacian of the weighted digraph $\Gamma$. (We recall that the weighted in-degree 
Laplacian $L$ for a digraph is defined as follows: for $i \neq j, L_{i j}=w_{j i}$ if $(j, i)$ is an edge and zero otherwise; meanwhile, $L_{i i}=-\sum_{j \neq i} L_{i j}$, see e.g. [19]). We remark that $L_{i j}$ captures the influence of component $j$ on component $i$, which is specified by the weight $w_{j i}$.

The described network model is well-known to achieve synchronization, i.e., the network components' states converge to a common value for any initial state $x[0]$, if 1 ) the digraph $\Gamma$ has a directed spanning tree (there is one vertex from which there is a directed path to every other vertex); and 2) $\sum_{i \in \mathbb{N}_{k}} w_{i k}<1$ for all vertices $k[22,23,24]^{2}$. Moreover, in this case, the synchronization law (the dependence of the synchronization value on the initial states) can be determined from the left eigenvector of $L$ associated with its dominant eigenvalue. We assume throughout our development that the nominal model satisfies these conditions and achieves synchronization.

Remark: State matrices of discrete-time synchronization models are often defined directly from an adjacency matrix, via normalization of each row to a unit sum (see e.g. [2]); it is easy to check that models of this type fall within the framework described here, and nominally satisfy the conditions for synchronization given connectivity. Thus, the results developed here carry through to processes defined in this way.

\section{Models for link failures:}

A subset of the network links are prone to failure. We call these critical links. Specifically, we consider the possibility that exactly one of $m$ critical links in this network process fails prior to the commencement of the synchronization process. We label the $m$ critical links as $\left\{l_{1}, l_{2}, \cdots, l_{m}\right\}$. Each link $l_{i}$ corresponds to the edge $\left(P_{i}, Q_{i}: w_{P_{i} Q_{i}}\right)$ in $\Gamma$. Failure of $l_{i}$ is modeled as setting the weight $w_{P_{i} Q_{i}}$ for term $\left(x_{P_{i}}-x_{Q_{i}}\right)$ to zero in Equation 1. That is the influence of the component $P_{i}$ on the evolution of $Q_{i}$ is removed. In graph-theoretic terms this can be viewed as the edge $\left(P_{i}, Q_{i}: w_{P_{i} Q_{i}}\right)$ being deleted from $\Gamma$ before the network process commences. We define the resulting graph as $\Gamma_{(i)}=\Gamma-\left(P_{i}, Q_{i}: w_{P_{i} Q_{i}}\right)$.

The evolution of the synchronization states across the network upon failure of the critical link $l_{i}$ is governed by:

$$
x[t+1]=A_{i} x[t],
$$

where $A_{i}=A_{0}-w_{P_{i} Q_{i}} e_{Q_{i}}\left(e_{P_{i}}-e_{Q_{i}}\right)^{\prime}$, and in general the notation $e_{q} \in \mathfrak{R}^{n}$ is used to represent a standard basis vector with entry $q$ equal to 1 .

We make the additional assumption the the graph $\Gamma_{(i)}$ still contains a directed spanning tree, which guarantees synchronization under the modified dynamics of Equation 3. The detection analysis can readily be adapted to the case where link failures break synchronization (in fact this is often easier since the dynamics change radically), but we exclude this possibility to provide a clearer and simpler analysis.

Remark: In many circumstances, all links may be prone to failure. This case is encompassed within our formulation, with all links being critical $(m=|E|)$. However, in many engineered networks, a priori knowledge may be available that only a subset of links are prone to failure (or are disproportionately likely to fail), due to knowledge about environmental impact factors, component stress, etc. In fact, in many cases, there

\footnotetext{
${ }^{2}$ The condition on the edge-weight sum is automatically satisfied if the discrete-time model is a sampling of a continuous-time process, and also is almost always a reasonable assumption for discretetime consensus algorithms.
} 
is a need to detect failure of a single susceptible link. To encompass these circumstances, we choose to distinguish critical links that are prone to failure. Specializations of the results to the important case with $m=|E|$ are remarked on at several points.

Observation Model

We consider a monitor that locally measures the network's dynamics in order to detect which (if any) of the critical links have failed. In general, these observation locations may be distant from the location of the failure in the network: the monitor must use such a remote signature to determine which critical link failure has occurred.

Formally, the monitor is modeled as making noisy measurements of the synchronization state at a single component $s$. The monitor's observations $y[t]$ are:

$$
y[t]=e_{s}^{\prime} x[t]+W[t]
$$

where the measurement noise $W[t]$ is assumed to be a zero-mean white Gaussian noise signal of intensity $\gamma^{2}$. The monitor is assumed to make measurements over the time interval $[0, T]$. We find it convenient to define an observation vector that stacks the monitor's measurements over the time horizon, i.e.

$$
Y_{T}=\left[\begin{array}{llll}
y[0] & y[1] & \cdots & y[T]
\end{array}\right]^{\prime}
$$

Remark: The technical development in this article is restricted to linear network processes and linear observations corrupted by Gaussian noise. Many complex network processes (e.g., power systems swing dynamics, oscillator circuits) are nonlinear. However, some of these processes admit linear approximations in typical operational regimes. In these cases, detectors can be built based on the linearizations, and the structural insights obtained here are can still inform sensor placement and detection. In a recent study, we have address line failure detection in the electric power network from phasor measurement unit data in this way, see [25].

\subsection{Detection Problem and Methodology}

We are interested in understanding if, and how accurately, the monitor can infer failure of a critical link from the remote observations $Y_{T}$. In particular, we are interested in understanding the role of the underlying network topology as well as observation location (component $s$ ) relative to the critical links in the monitor's ability to detect the failure. The detection problem depends on the monitor's model for possible failures (here assumed to be a known probabilistic model) as well as the initial state $x[0]$. In our analysis, we first consider the case that the monitor knows the initial state $x[0]$ exactly (which we refer to as known-state link failure detection, or KS-LFD). Then, we consider the case that the initial state is unknown to the monitor and modeled as Gaussian random variable $x[0] \sim \mathcal{N}\left(0, \sigma^{2} I\right)$ which is independent of the measurement noise. We refer to this case as stochastic-state link failure detection, or SS-LFD.

It is worthwhile to discuss applications of each problem. The SS-LFD problem arises more commonly in real-world health-monitoring and security problems for networks, since the monitor often will only have knowledge of local states and dynamics. However, KS-LFD is apt in certain special circumstances: for instance, when the detection task is being conducted as a post-processing step, if a test case is deliberately being used 
for detection, or historical knowledge provides insight into typical initial states of the network components. As an example, for a Wireless Sensor Network (WSN) operating in a harsh environment, a manager may deliberately apply a test involving a local state perturbation to evaluate network health. In this case, the initial state will be known, as an almost-synchronized state with only a deliberate local disruption. Meanwhile, for infrastructural applications (e.g., fault localization in electric power networks), typical pre-fault operating conditions of the network may sometimes be known, especially for a post-analysis. In addition to these explicit uses, the analysis of KS-LFD serves as a stepping stone for the analysis of SS-LFD. For real-time detection, the unknown initialstate case is often more apt.

\section{Detection Methodology:}

The monitor's detection performance will depend on the particular detection scheme used. A natural approach is to cast the problem as a classical hypothesis testing problem, and employ an optimal detection scheme. In this work, we consider the performance of a Maximum A Posteriori Probability (MAP) detection scheme. Next let us pose the link-failure detection problem as a MAP detection problem.

For both the KS-LFD and SS-LFD problems, the network monitor's goal is to decide between $m+1$ hypotheses. Specifically, using the data sequence $Y_{T}$, the monitor must distinguish between the following:

$$
\begin{aligned}
& H_{0}:=\text { None of the critical links have failed } . \\
& H_{i}:=\text { Link } l_{i} \text { has failed. } \forall i=1,2, \cdots, m .
\end{aligned}
$$

Hypothesis $H_{0}$ corresponds to the nominal case that no link failures have occurred, and is referred to as the null hypothesis per standard detection-theory terminology. Each hypothesis is modeled as having a known a priori probability of occurring. We denote these probabilities as $\operatorname{Pr}\left(H_{i}\right)=p_{i}$ for $i=1, \cdots, m$, and $\operatorname{Pr}\left(H_{0}\right)=1-\sum_{k=1}^{m} p_{k}=p_{0}$. Conceptually the a priori probability $p_{i}$ reflects the monitor's initial belief in the susceptibility of link $l_{i}$ to failure; a higher $p_{i}$ represents a more susceptible $l_{i}$. In seeking to distinguish between the hypotheses, it is assumed that the monitor has knowledge of the nominal network topology and the link failure model. In other words, it is assumed that the monitor knows the nominal synchronization process model $\left\{A_{0}\right\}$ and the critical links $\left\{l_{1}, l_{2}, \cdots, l_{m}\right\}$.

The MAP detection scheme employs a Bayesian approach to choose the hypothesis with the highest a posteriori probability given the observation vector $Y_{T}$. Specifically, the monitor's selection $\tilde{H}$ is the hypothesis $H_{i}$ if

$$
\operatorname{Pr}\left(H_{i} \mid Y_{T}\right) \geq \operatorname{Pr}\left(H_{j} \mid Y_{T}\right) \quad \forall j \in 0, \cdots, m
$$

The detection scheme in general is imperfect, i.e. the monitor may choose hypothesis $H_{i}$ while in reality hypothesis $H_{j}$ occurred. It is natural to consider the probability of error for the detector, which is equal to

$$
P_{\text {err }}=\sum_{i} \operatorname{Pr}\left(\tilde{H} \neq H_{i} \mid H_{i}\right) p_{i},
$$

as a measure of the detector's performance. In particular, we define three notions of the performance for the MAP detector, as follows. 
- Undetectability: We consider the link failure to be undetectable to the MAP detection scheme if the a posteriori probability of each hypothesis $H_{i}$ equals the a priori probability, i.e. $\operatorname{Pr}\left(H_{i} \mid Y_{T}\right)=p_{i}$ for all $i=0, \cdots, m$ regardless of the observation window $[0, T]$. In this case, the probability of error $P_{\text {err }}$ of the MAP detector is equal to the a priori probability of error $1-\max _{i \in(0, \cdots, m)} p_{i}$.

- Improved Detection: We say that improved detection of the link failure is achieved if the MAP detector's probability of error is strictly less than the a priori for some observation window $\left(0 \leq P_{\text {err }}<1-\max _{i \in(0, \cdots, m)} p_{i}\right.$ over some window $\left.[0, T]\right)$.

- Asymptotic Perfect Detection: A special case of improved detection is that the error probability approaches zero with sufficient observation horizon, i.e. $P_{\text {err }} \rightarrow 0$ as $T \rightarrow \infty$. We refer to this case as asymptotic perfect detection.

We note that the MAP scheme is efficient, in the sense that it achieves the minimum probability of error among possible detectors.

Remark 1. The a priori failure probabilities of the critical links are assumed known in the formulation above. In some real-world examples, this information can be extracted from historical data, or an understanding of the underlying network's development (e.g., ages of components, etc). However, other applications do not naturally permit modeling and analysis of a priori component-failure probabilities. In cases where the a priori probabilities are unknown, the optimal detection problem is typically phrased in terms of the maximum probability of misdetection among the hypotheses, i.e. $\max _{i} \operatorname{Pr}(\tilde{H} \neq$ $H_{i} \mid H_{i}$ ) (where the maximum may be computed over all hypotheses or all hypotheses other than the null hypothesis). Specifically, an optimal detector is one that minimizes this maximum probability. For the class of problems studied here, the optimal detector for this problem can be equivalenced with the optimal detector in our formulation, for the special case that the prior probabilities are identically $\frac{1}{m+1}$ (or the priors for all hypotheses other than the null hypothesis are identical). We will revisit this special case in our presentation of results.

Remark 2. The probability-of-error calculation can naturally be phrased using the classical hypothesis testing terminology such as size, power, and identifiability. The focus of this article is on understanding the role of the network's graph topology on detection rather than the statistical aspects, so we do not develop the hypothesis-testing formalisms in detail.

\section{MAP Detection: Known Initial State}

The goal of this section is to analyze the form and performance of the MAP linkfailure detector from a graph-theoretic perspective, KS-LFD problem. This analysis addresses the core question of whether remote local response signatures are sufficient for identifying link failures. The graph-theoretic analysis also gives insight into effective monitoring locations, which may be useful for sensor placement, network protection from intrusion, evaluation of stakeholder information content, etc. Toward developing the graph-theoretic results, algebraic expressions for the hypothesis test and error are 
first obtained (Section 3.1), using standard hypothesis-testing constructs. These expressions are then used to obtain matrix-theoretic and spectral characterizations of the performance, and in turn graphical analyses (Section 3.2).

Several of our results are phrased in terms of the observability matrices of the network model under each hypothesis:

$$
O_{i}(T)=\left[\begin{array}{c}
e_{s}^{\prime} \\
e_{s}^{\prime} A_{i} \\
\vdots \\
e_{s}^{\prime} A_{i}^{T-1}
\end{array}\right] \quad \forall i=0,1, \cdots, m
$$

where $s$ is the observation location while $i$ indexes the hypothesis. We recall that the observability matrix for a linear system indicates whether the system's initial state can be reconstructed from its observation sequence over an interval (i.e., whether the system is observable). Full column rank of the observability matrix is necessary and sufficient for the system to be observable. Our analyses also draw on the notion of observability for a mode or eigenvalue of a linear system, which captures whether the state projection in the modal direction can be reconstructed. The reader to [26] and [27] for general conditions for modal observability. For the dynamics considered here (Equations 2 and 3 ), it is easy to check that any non-repeated eigenvalue $\lambda_{l(i)}$ of $A_{i}$ is observable if $e_{j}^{\prime} v_{l(i)} \neq 0$, where $v_{l(i)}$ is the right eigenvector associated with $\lambda_{l(i)}$ (which is unique to within a scaling).

\subsection{Preliminary Results}

For the KS-LFD problem, the observation vector $Y_{T}$ is a Gaussian random vector under each hypothesis:

$$
f_{Y_{T} \mid H_{i}}=\left|(2 \pi)^{T} \Sigma_{i}\right|^{-\frac{1}{2}} \exp \left[-\frac{1}{2}\left(Y_{T}-\mu_{i}\right)^{\prime} \Sigma_{i}^{-1}\left(Y_{T}-\mu_{i}\right)\right]
$$

where

$$
\begin{aligned}
\mu_{i} & =E\left(Y_{T} \mid H_{i}\right)=O_{i}(T) x[0] \\
\Sigma_{i} & =\operatorname{Cov}\left(Y_{T} \mid H_{i}\right)=\gamma^{2} I
\end{aligned}
$$

The MAP detector can be implemented by sequentially comparing posterior probabilities of pairs of hypotheses and finally choosing the one with highest probability. This reduces to a linear test on the observations because the posteriors are jointly Gaussian [28]. The probability of error admits an exact calculation in the case with two hypotheses. In the multi-hypothesis test, an exact computation becomes cumbersome because the Gaussian distribution must be integrated over a piecewise-defined region in space. A number of good bounds are available; a simple one is sufficient for our purposes here. The sequential comparison of hypotheses and the computation/bound of $P_{\text {err }}$ can expressed as follows:

Lemma 1. For the KS-LFD problem, the decision rule used by MAP detector to compute the final decision $\tilde{H}$ is given by:

$$
Y_{T}^{\prime}\left(\mu_{i}-\mu_{j}\right) \underset{\tilde{H} \neq H_{i}}{\stackrel{\tilde{H} \neq H_{j}}{\gtrless}} \frac{1}{2}\left(\mu_{i}^{\prime} \mu_{i}-\mu_{j}^{\prime} \mu_{j}\right)+\gamma^{2} \log \frac{p_{j}}{p_{i}}, \quad \forall H_{i}, H_{j},
$$


where $\mu_{i}=O_{i}(T) x[0]$ and $\mu_{j}=O_{j}(T) x[0]$.

Furthermore, the probability of error in detection $P_{\text {err }}$ satisfies:

$$
P_{\text {err }} \leq \frac{m}{2}-\frac{1}{2} \sum_{i=0}^{m} p_{i} \sum_{j \neq i} \operatorname{erf}\left(\frac{\left\|\mu_{i}-\mu_{j}\right\|_{2}}{2 \gamma}+\frac{\gamma \log \frac{p_{i}}{p_{j}}}{\left\|\mu_{i}-\mu_{j}\right\|_{2}}\right)
$$

where, the standard error function $\operatorname{erf}(x)$ is defined as $\frac{2}{\sqrt{\pi}} \int_{0}^{x} e^{-z^{2}} d z$. This expression for the error probability is exact for the case of two hypotheses, $m=2$.

The statements in Lemma 1 follow directly from classical results on the hypothesis test with Gaussian observations [28], so details are omitted. We remark here that the detector chooses among the $(m+1)$ hypotheses according to an $(m+1)$-way partition of the observation space, which is specified by the intersections of the hyper-spaces in Equation 9. The dividing hyper-planes pass through the origin.

The vectors $\mu_{i}-\mu_{j}$, which we call the differential signature of hypothesis $H_{i}$ over hypothesis $H_{j}$, capture the differences between the monitor's expected observation sequence for each hypothesis for a given initial state. It is easy to verify that the probability of error is monotonic in the energy: the larger the energy (norm) of the differential signature of each pair of failures, the smaller the confusion probability between the two hypotheses.

To continue our analysis of the performance of the MAP detection scheme, we rely on an expansion of the term $e_{s}^{\prime}\left(A_{i}^{k}-A_{j}{ }^{k}\right)$, which appears as rows of $O_{i}-O_{j}$. The expansion captures the mismatch in the network's dynamics between the two hypotheses and allows us to develop an alternate expression for the differential signature, as well as conditions for undetectability. The term can be expanded as follows (see the Appendix for the a short proof)

$$
e_{s}^{\prime}\left(A_{i}^{k}-A_{j}^{k}\right)=\sum_{p=1}^{k} e_{s}^{\prime} A_{i}^{k-p}\left(A_{i}-A_{j}\right) A_{j}^{p-1} \quad \forall k \geq 1
$$

Using Equation 11, we obtain an alternate expression for the differential signature $\mu_{i}-\mu_{j}$, and its energy. Specifically, we show that the differential signature can be decomposed in terms of convolutions of two signals: 1) an initial-state response of a faulted system across a particular critical edge; and 2) a propagative response seen by the monitor (for a faulted system) due to a state perturbation at the trailing edge of a failed link. Here is the formal result.

Theorem 1. (Energy of a Differential Signature) Consider the differential signature of hypothesis $H_{i}$ over $H_{j}$, namely $\mu_{i}-\mu_{j}$. The energy of this signal is given by:

$$
\begin{aligned}
\left\|\mu_{i}-\mu_{j}\right\|_{2}^{2} & =\sum_{k=0}^{T}\left[\left(r_{(i, j)} * z_{(j, j)}\right)[k]-\left(r_{(i, i)} * z_{(i, j)}\right)[k]\right]^{2} \quad i, j \neq 0 \\
\left\|\mu_{i}-\mu_{0}\right\|_{2}^{2} & =\sum_{k=0}^{T}\left[\left(r_{(i, i)} * z_{(0, i)}\right)[k]\right]^{2} \quad i \neq 0
\end{aligned}
$$

where $\left(r_{(a, b)} * z_{(d, f)}\right)[k]=\sum_{p=0}^{k-1} r_{(a, b)}[p] z_{(d, f)}[k-p]$ is the convolution of $r_{(a, b)}$ and $z_{(d, f)}$. Here,

$$
r_{(a, b)}[l]=\left\{\begin{array}{lc}
\left(e_{P_{b}}-e_{Q_{b}}\right)^{\prime} A_{a}{ }^{l} x[0] & \text { if } l=0, \ldots, T-1 \\
0 & \text { otherwise }
\end{array}\right.
$$


and

$$
z_{(d, f)}[l]=\left\{\begin{array}{lc}
w_{P_{f}, Q_{f}} e_{s}^{\prime} A_{d}{ }^{l} e_{Q_{f}} & \text { if } l=0, \ldots, T-1 \\
0 & \text { otherwise }
\end{array} .\right.
$$

The theorem exposes that two factors combine to determine failure's signature at the monitoring location: the nominal initial-state response at the potential failure location, and the propagative response at that monitor due to a disturbance at that failure location.

Remark: The case where all liks are prone to failure and the a priori failure probabilities are unknown is of special interest. The optimal (minimax) detector in this case can be obtained by setting $m=|E|$ and setting the probabilities of the hypotheses to be equal. The detector form and probability of error bound simplify in this case. Specifically, the term $\gamma^{2} \log \frac{p_{j}}{p_{i}}$ drops out of the comparison of the hypotheses, which then yields an error probability bound that depends only on terms like erf $\left(\frac{\left\|\mu_{i}-\mu_{j}\right\|_{2}}{2 \gamma}\right)$. We note that the error probability in this case can also be interpreted as the minimax confusion probability of the optimal hypothesis test.

\subsection{Performance of MAP Detector}

The preliminary algebraic results allow us to characterize the performance of the MAP detector. We begin with several easy-to-test matrix-theoretic and spectral conditions for undetectability, in Theorem 2.

Theorem 2. For the KS-LFD problem, the following are conditions for undetectability of critical-link failures:

1. The link failure is undetectable regardless of initial state $x[0]$ if and only if all the columns of $\left(A_{0}-A_{i}\right)$ are in the null space of the observability matrix $O_{0}(T)$ for every hypothesis $H_{i}(i>0)$.

2. Assume that all of the Jordan blocks of $A_{0}$ have size 1 . The critical-link failures are undetectable if for every observable eigenvalue of $A_{0}$, the $Q_{i}{ }^{\text {th }}$ entry of the corresponding left eigenvector is zero. Conversely, if for some critical link $l_{i}$, there is at least one observable eigenvalue whose associated left eigenvector has a non-zero $Q_{i}{ }^{t h}$ entry, then the probability of error in the detection improves for all generic initial states.

3. The link failures are undetectable only if the observable eigenvalues are insensitive to every possible link failure.

Remark: In the above theorem, the spectral condition for undetectability has been presented for non-defective $A$, for convenience. If $A$ is defective (has Jordan blocks of size greater than 1), then a spectral condition for undetectability can still be developed, but must be phrased in terms of the observable subspace associated with each eigenvalue, and the projections of the corresponding left eigenspaces into the observable subspace. A careful presentation of the result requires some technical machinery related to generalized eigenvectors, and hence is omitted.

An important outcome of Theorem 2 is that the condition for undetectability of the link failures is typically much stronger than the condition for the system-theoretic notion of unobservability. Unobservability of the linear system $\left(A_{0}, e_{j}^{\prime}\right)$ only requires that $O_{0}(T)$ 
has a null space, while undetectability requires that the difference between the nominal and faulted network matrices have all columns in this null space, for all possible failures (from Statement 1). Equivalently, Statement 2 shows that undetectability can result from a generic (known) initial condition only if all modes of the system are unobservable, while unobservability only requires a single mode to be unobservable. Thus we see that improved detection of link failures is possible under broader conditions than that are needed for observability.

Theorem 2 serves as a starting point for a graph-theoretic characterization of MAP detector's performance. The following graph-theoretic result relates the presence/absence of paths between the observation and link-failure locations to detectability/undetectability. The essence of the result is that improved detection is achieved whenever there is a directed path from the trailing end of a critical link to the observation location for generic $x[0]$. These directed paths are sufficient for transmitting information about the criticallink characteristic to to MAP detector. Here is the result.

Theorem 3. For arbitrary known $x[0]$ and the observation horizon $[0, T]$, the link failure is undetectable if and only if there are no directed paths in $\Gamma$ to component $j$ (observation location) from the trailing ends of every critical link. Otherwise, the probability of error in the detection improves for a generic initial state.

Furthermore, if improved detection is possible, the MAP detection scheme improves upon the a priori probabilities for an observation horizon of $[0, T]$, if and only if $T \geq T_{\min }$ where $T_{\min }$ is the distance (shortest length of a directed path) to the observation location from the trailing end of a critical link.

This key result shows that remote monitoring facilitates detection of link failures, as long as there is a path from the failure location to the monitor. This is in contrast with graph-theoretic conditions for observability. Existing literature has shown that unobservability can occur even in strongly connected graphs, due to e.g. symmetry structures $[29,30]$. We stress that that the condition for improved detection is independent of the number of critical links, hence it holds true whether ev

In the case that improved detection is possible, it is natural to study whether or not perfect detection is possible. In the next theorem, we present an algebraic condition under which the MAP detector can perfectly distinguish between the $m+1$ hypotheses, when the observation horizon is made sufficiently long. Further, for generic initial state $x[0]$, we provide a broad graph-theoretic condition for asymptotic perfect detection for the link-failures. In particular, we show that strong connectedness allows asymptotic perfect detection.

Theorem 4. Consider the link-failure detection problem. Let $v_{1(i)}=\mathbf{1}$ be the dominant right eigenvector of $A_{i}$ (hypothesis $H_{i}$ ) associated with the unity eigenvalue. Further let $w_{1(i)}$ be the corresponding left eigenvector such that $\left\|w_{1(i)}\right\|_{1}=1$.

The probability of error in link-failure detection, $P_{\text {err }}$, approaches 0 as $T \rightarrow \infty$, if and only if $w_{1(i)}^{\prime} x[0] \neq w_{1(j)}^{\prime} x[0]$ for every pair of hypotheses $H_{i}$ and $H_{j}$, i.e. the asymptotic synchronization state is distinct under each hypothesis.

Furthermore, for a generic initial state $x[0]$, strong connectedness of the network graph for the nominal synchronization dynamics (hypothesis $H_{0}$ ) is sufficient to ensure asymptotic perfect detection. 
The connection between these results and the conditions for "hearing" a network are interesting [20]. From Theorem 2, we see that detectability crucially depends on whether a link failure changes any of the eigenvalues. Considering this result together with the graph-theoretic conditions for detectability (Theorems 3 and 4), it then becomes apparent that each possible failure configuration of the network maps to a different spectra, and hence each is distinguishable provided that there is a path from the failure to the monitoring location. Thus, while it is generally not possible to "hear" the network just from its eigenvalues [20], we find that it is possible distinguish between a finite set of network topologies corresponding to possible link failures, and to do so using local measurements.

When perfect detection is possible asymptotically, the rate at which the error probability approaches 0 can also be characterized:

Theorem 5. If asymptotic perfect detection can be achieved, then the error probability is upper bounded by a geometric function of the measurement horizon T: $P_{\text {err }} \leq \alpha \eta^{T}$, for some $\alpha>0$ and some $\eta \in(0,1)$.

Some discussion about the results on asymptotic perfect detection are necessary. First, we stress that the result assumes a generic initial state $x[0]$ : certain specific initial states can prevent $P_{\text {err }}$ from approaching zero asymptotically. Second, we note that asymptotically perfect detection is connected to the consistency of Maximum Likelihood (ML) estimators. To see this, we note that the synchronization process reaches an asymptote, whereupon the measurement process under each hypothesis consists of independent samples from a fixed distribution. ML-estimator consistency follows assuming that these distributions are different for any two hypotheses. In other words, asymptotic perfect detection and ML-estimator consistency both lead to the condition that the synchronization values are distinct under each hypothesis under generic initial state.

Asymptotically-perfect detection is centrally related to whether or not the link failure alters the synchronization rule, Equation B.6. For a strongly connected network graph, asymptotically-perfect detection is achieved (for generic initial states) since the synchronization value, or equivalently the dominant left-eigenvector, always changes after a critical link has failed. For other classes of graphs satisfying the conditions in Section 2, asymptotically-perfect detection may or may not be achieved, depending on whether or not the initial state is in the null space of $w_{(i)}^{\prime}-w_{(j)}^{\prime}$. It is worth stressing that the class of graphs for which asymptotically-perfect detection is achieved is quite broad (including all strongly-connected graphs), and only very special circumstances permit detection but not asymptotically-perfect detection. In sum, in the known-initial-condition case, connectivity of the network is sufficient not only for detectability (improved detection) but also for asymptotically perfect detection: remote signatures can be used for detecting local structural changes.

Remark: The exponential bound on the error probability immediately allows analysis of the time required to detect the failure with a certain fidelity. In particular, achieving an error probability of $\bar{p}$ requires a time horizon of $T \geq \frac{\ln (\bar{p})-\ln (\alpha)}{\ln (\eta)}$, where $\alpha$ and $\eta$ are as given in Theorem 5 . The required time shows a strong inverse dependence on $\eta$. From the proof of the theorem, we find that $\eta$ is governed by $\exp \left(-\left(\min _{i, j}\left(w_{1(i)}^{\prime}-w_{1(j)}^{\prime}\right) x[0]\right)^{2}\right)$, where the minimum is taken over all pairs of hypotheses. Thus, we see that it is the 
change in the steady-state synchronization value resulting from each possible line failure that determines the detection time: the more the change, the faster the detection.

Remark: The asymptotic analysis of the detector's performance allows an interesting observation regarding the information needed by the detector. In general, the MAP detection scheme requires knowledge of the full synchronization model. Asymptotically, however, the detector simply differentiates between the steady-state synchronization value for each hypothesis, and further this differentiation is sufficient for perfect asymptotic detection. Thus, effective detection can be achieved simply from knowledge of the steadystate synchronization law for each hypothesis. The time horizon required until the the asymptotic model is in force depends on the settling characteristics of the synchronization model. This settling time can be bounded in terms of the eigenvalues of the state matrix, but in general depends in a complicated way on the full structure of the network model (see e.g. [19]).

Remark: The developed method is scale free, in the sense that a single observation permits perfect detection asymptotic detection regardless of the network size. The measurement horizon needed for detection does depend on the network size and the locations of the critical links relative to the observer. However, the required time generally scales gracefully with the network size.

\section{MAP Detection: Unknown Initial State}

In this section, matrix- and graph- theoretic characterizations results are developed for the LFD-SS problem. The algebraic analysis for this case becomes more intricate: an algebraic expression for the hypothesis test can be obtained, but the probability of error becomes difficult to compute explicitly, even in the case of two hypotheses. However, by using the known-initial-state case as a stepping stone, we are able to develop graph-theoretic necessary and sufficient conditions for detectability/undetectability, and to characterize perfect detection for the stochastic initial state case. Additionally, formal expressions for the error can be exploited to develop upper bounds, using the Chernoff inequality.

\subsection{Algebraic Preliminaries}

To begin, let us pursue an algebraic characterization of the MAP detector for the random-initial-state case. From the assumption of Gaussianity for $x[0]$ and $W[t]$, it follows that the observation vector $Y_{T}$ is a Gaussian random vector under each hypothesis. In particular, we observe that:

$$
f_{Y_{T} \mid H_{i}}=\left|(2 \pi)^{T} \Sigma_{i}\right|^{-\frac{1}{2}} \exp \left[-\frac{1}{2} Y_{T}^{\prime} \Sigma_{i}^{-1} Y_{T}\right]
$$

where $\Sigma_{i}$ (as defined above) is the covariance matrix $\operatorname{Cov}\left(Y_{T} \mid H_{i}\right)$.

Working from the general MAP detection framework (Equation 6) and the density function of $Y_{T}$ (Equation 13) we can simplify the decision rule for the MAP detector to a sequence of comparisons as follows. 
Lemma 2. The MAP hypothesis $\tilde{H}$ can be obtained by applying the following decision rules sequentially

$$
\frac{1}{2} Y_{T}^{\prime}\left(\Sigma_{i}^{-1}-\Sigma_{j}^{-1}\right) Y_{T} \underset{\tilde{H} \neq H_{i}}{\stackrel{\tilde{H} \neq H_{j}}{\gtrless}} \log \left(\frac{p_{j}}{p_{i}}\right)+\frac{1}{2} \log \left|\Sigma_{i} \Sigma_{j}^{-1}\right|
$$

where have we defined $\Sigma_{i}=\sigma^{2} O_{i}(T) O_{i}^{\prime}(T)+\gamma^{2} I$ for each hypothesis $H_{i}$,

The statements in Lemma 2 follow directly from the classical hypothesis test with Gaussian observations with density given by (13), so details are omitted. The detector for a random-initial-state case involves a quadratic function of the observation sequence. Specifically, the value of the multi-dimensional hyperboloid $Y_{T}^{\prime}\left(\Sigma_{i}^{-1}-\Sigma_{j}^{-1}\right) Y_{T}$ in the observation space determines which hypothesis is selected. Noting that the symmetric matrix $\left(\Sigma_{i}^{-1}-\Sigma_{j}^{-1}\right)$ may or may not be definite, the hyperboloid may define either a closed or an open surface (see [31]).

\subsection{Performance of MAP Detector}

We begin our analysis of the MAP detector's performance with an algebraic conditions for undetectability. Undetectability results if the terms associated with the observations in Equation 14 vanish, as formalized in the following theorem:

Theorem 6. Consider the SS-LFD problem. The link failures are undetectable (i.e., $M A P$ detection from the observation vector $Y_{T}$ does not reduce the a priori error probability) if and only if $\Sigma_{0}=\Sigma_{i}$ for all $i=1, \cdots, m$.

Next, we develop a graph-theoretic condition under which the critical link failures are undetectable regardless of the observation window $[0, T]$, which turns out to be exactly the same as for the KS-LFD problem.

Theorem 7. Consider the SS-LFD problem. The link-failure is undetectable regardless of the observation horizon, if and only if there is no directed path in $\Gamma$ to the observation location $j$ from the trailing end $Q_{i}$ of each critical link $l_{i}$.

The necessary and sufficient graph-theoretic conditions for the SS-LFD and KS-LFD problems are identicial. Therefore, we can establish direct equivalence between the various conditions for undetectability under both the cases. Specifically, the algebraic conditions on $\operatorname{Cov}\left(Y_{T} \mid H_{i}\right)$ in Theorem 6 are identical to the conditions on the null space of $O(T)$, and to the spectral condition on $A$ in Theorem 2. Thus, by using the known initial state case as a stepping-stone, we are able to obtain easy-to-test algebraic, graphtheoretic, and spectral conditions for undetectability when the initial state is unknown.

Although the conditions for undetectability are identical under the two cases, the performance of the MAP detector for the unknown-initial-state case cannot be better than the known-initial-state case. Let us discuss the performance of the detector further, by characterizing the probability of error in detection for the unknown-initial-state case.

In contrast with the known-initial-state case, the detector form for the random-initialstate case does not readily permit explicit computation of the detection error probability, nor can it be bounded simply. This complication stems primarily from the quadratic form in Equation 14, which results in error distributions that are not Gaussian. However, we can develop bounds on the probability of error using Markov's and Chernoff's inequalities for non-negative random variables as formalized in the following result. 


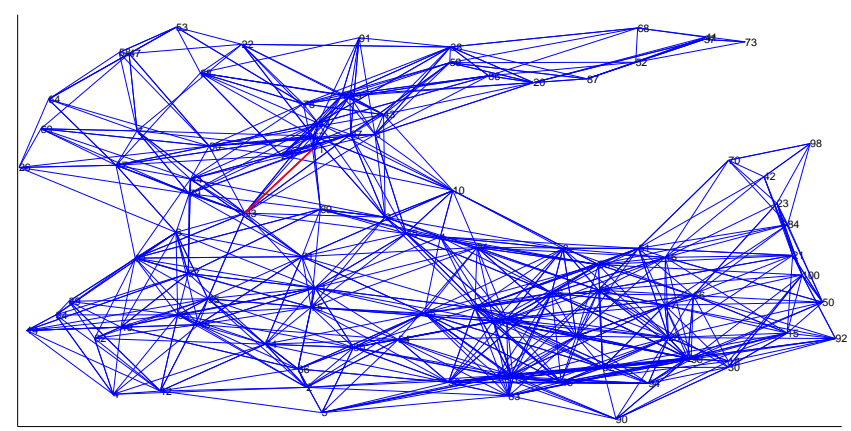

Figure 1: Nominal network for KS-LFD problem. The ten most effective nodes for measurement are also shown.

Theorem 8. For the SS-LFD problem, the probability of error can be bounded as follows.

$$
P_{\text {err }} \leq \min \left(1-\max _{i}\left(p_{i}\right), \sum_{i=0}^{m} \min \left[1,\left(\inf _{\bar{s}} e^{\bar{s} \zeta} \theta_{i, j}(-\bar{s})\right)\right] p_{i}\right)
$$

where $\theta_{i, j}(\bar{s})$ is the moment generating function of the random variable

$$
Y_{T}^{\prime}\left(\Sigma_{i}^{-1}-\Sigma_{j}^{-1}\right) Y_{T}
$$

conditioned on $H_{i}$.

Remark: The conservativism of the bound has been explored in the context of several simulation examples. These examples indicate that the error bound scales proportionally with the error, as model parameters are changed (e.g., measurement noise, variability of the initial condition, network size, etc).

Beyond developing upper bounds, one can give more precise characterizations of the error probability in some limiting cases. In contrast with the known-initial-state case, the error probability does not approach zero with growing measurement horizon under broad conditions. In other words, MAP detection scheme for unknown-initial-state case typically leads to improved detection but not asymptotic perfect detection. It can be shown that the asymptotic error probability becomes diminishingly small with decreasing measurement noise, whenever the conditions for detectability are met. Also, noticing that detectability is commensurate with statistical identifiability, the error probability alternately could be made small through use of repeated trials. Details are omitted.

\section{Illustrative Examples}

Two numerical examples are given to illustrate the solution of the KS-LFD and $S S$ LFD problems, and to give some insights into sensor placement for failure detection.

\subsection{Example: KS-LFD}

A network with 100 nodes is considered. The (weighted, directed) graph $\Gamma$ for the network model was generated as follows: vertices were placed uniformly within the unit 


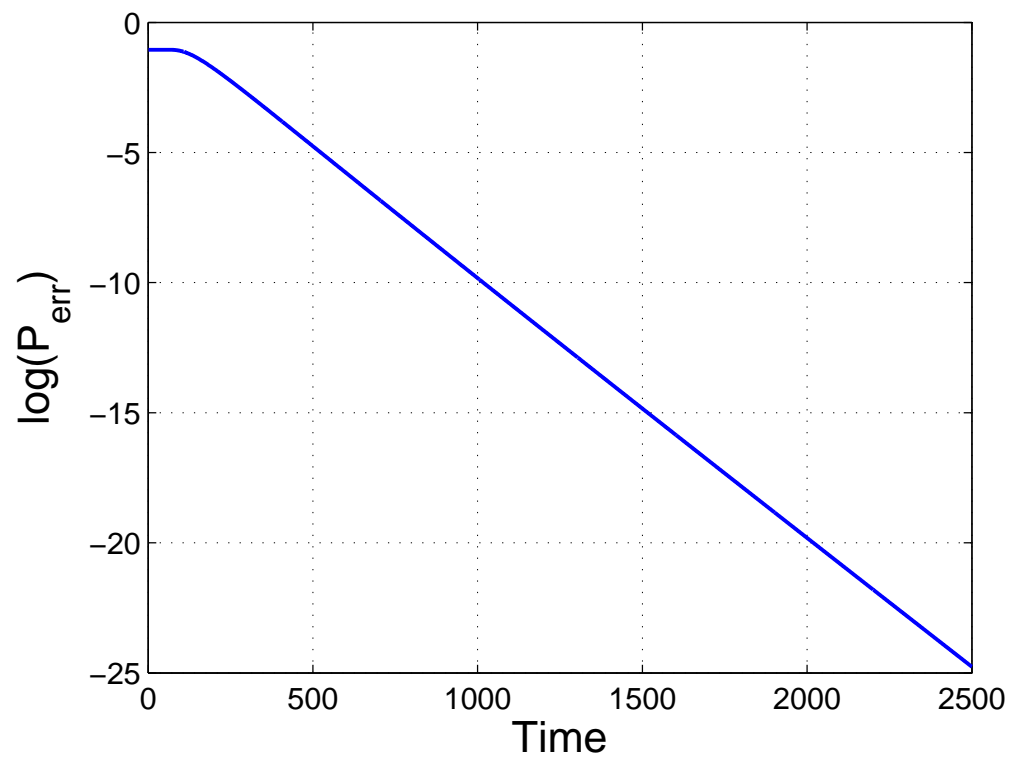

Figure 2: Probability of error in a logarithmic scale.

square, and edges were drawn in both directions between any two vertices that were sufficiently close; the weights of the directed edges were chosen independently and uniformly on $[0,1])$. The process of forming a network graph replicates connectivity structures that are observed in terrestrial networks, which include strongly-connected subnetworks with weak links between them. The graph is shown in Fig. 1. Per the problem formulation described in Section 2, a linear synchronization process among the network components is considered (Equation 2). The link from node 71 to node 93 (highlighted in red) is assumed to be susceptible to failure. Specifically, this particular link can fail with a probability of 0.65 (Equation 3). The initial state of the synchronization process is randomly generated, but assumed known to the monitor (as may be the case in a post-processing scenario, or for a deliberate test of the network).

We first consider a monitor that makes noisy measurements of the local state at single node, in this case node 56. Specifically, the measurements are corrupted by zero-mean white Gaussian noise with intensity $\gamma^{2}=0.1$ (Equation 4). We consider MAP detection of the link failure (Section 2.2). Fig. 2 shows the error probability of the link-failure detector as a function of the observation horizon on a logarithmic scale. The plot shows that the probability of error is asymptotically approaching zero exponentially, which is expected per Theorem 4 and 5.

In this numerical example, we also compare the detector's performance for different measurement locations, to gain a preliminary understanding of sensor selection for linkfailure detection. Specifically, for observations at each network node, we determine the time horizon required to achieve a probability of less than $10^{-5}$ (see Fig. 3). In Fig. 1, we have highlighted the ten nodes in the network at which the fewest observations are needed for detection. Our results verify that, for every location from which the link failure is detectable, the MAP scheme perfectly detects the link failure asymptotically. We see that 
detection is fastest when observations are made at the location of the link failure (node 71). We also stress that effective detection is achieved with a relatively small observation horizon, regardless of the measurement location.

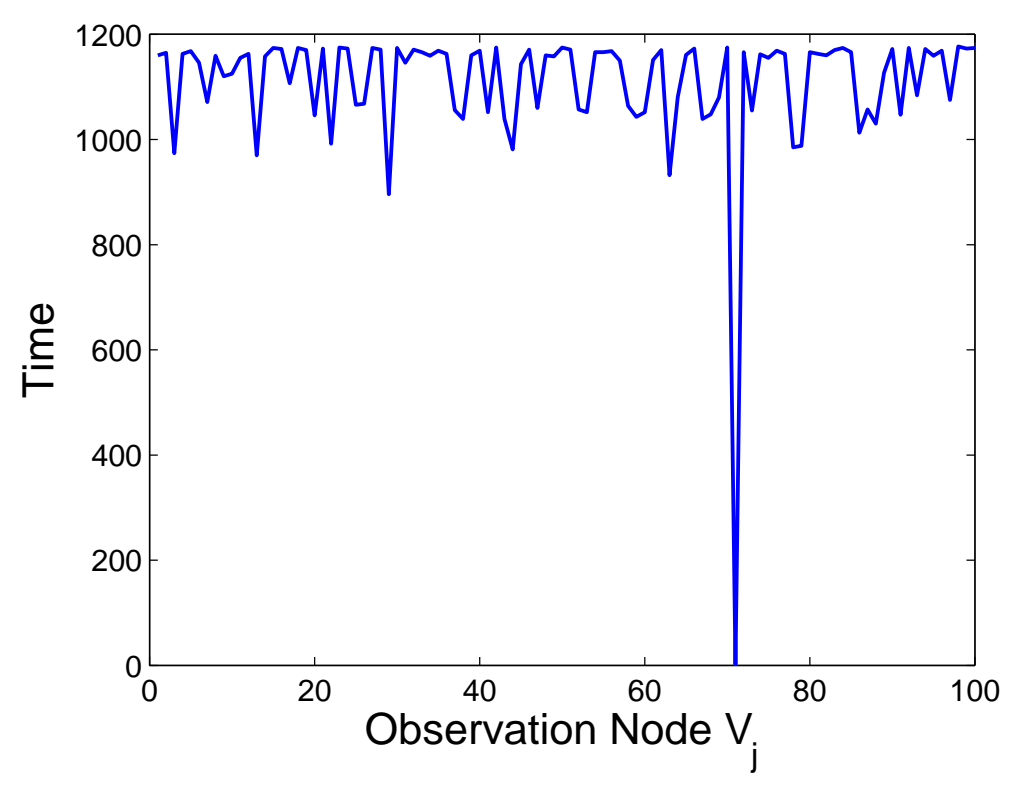

Figure 3: Observation Location vs Time till perfect detection

The example illustrates that fast detection is achieved when the observation location is near the link failure in the graph.

\subsection{Example: $S S-L F D$}

The same network with 100 nodes is used to illustrate SS-LFD. The network graph is shown in Fig. 4. The initial state of the network is unknown, and assumed to be modeled by a Gaussian random variable with zero mean and a covariance matrix $\sigma^{2} I$, i.e. $x[0] \sim \mathcal{N}\left(0, \sigma^{2} I\right)$, where $\sigma^{2}=50$. For the purposes for our simulation, a monitor is assumed to make noisy observations at different nodes. The measurements are corrupted by a zero-mean Gaussian noise of intensity $\gamma^{2}=1 E-8$ (Equation 4). Fig. 5 compares the probability of error as a function of the measurement window for two observation locations (node 1 and node 10). The plot illustrates that the MAP detection scheme is able to reduce the initial probability of error significantly for both observation locations, however the detection scheme is imperfect asymptotically. Fig. 6 illustrates the time taken for the MAP detector to be within 1 percentage point of the steady-state probability of error: faster detection is achieved near the link failure, at the edges of the network, and at some strongly connected nodes that are partitioned from the failure location. Fig. 7 shows how the the steady-state value of $P_{\text {err }}$ depends on the measurement location: the steady-state errors follow a similar pattern to the detection time.

As a demonstration of the conservatism bound on $P_{\text {err }}$ for the SS-LFD case (Theorem 8, we compared the actual $P_{\text {err }}$ against the bound for different noise levels for a small 5 


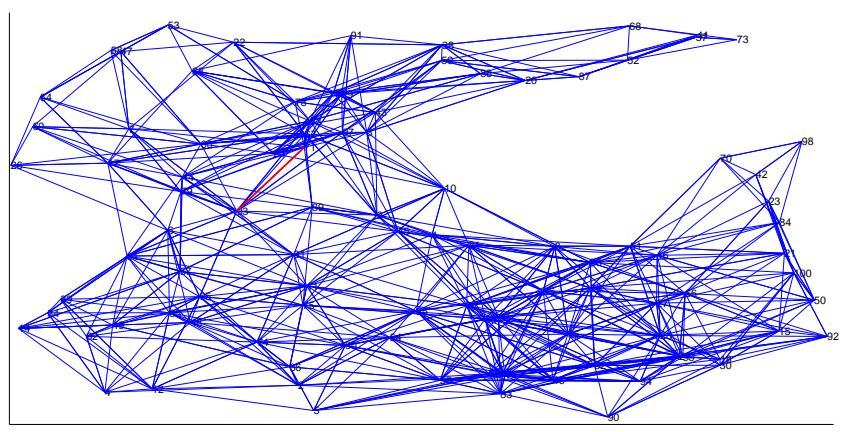

Figure 4: Network for the unknown $x[0]$ case.

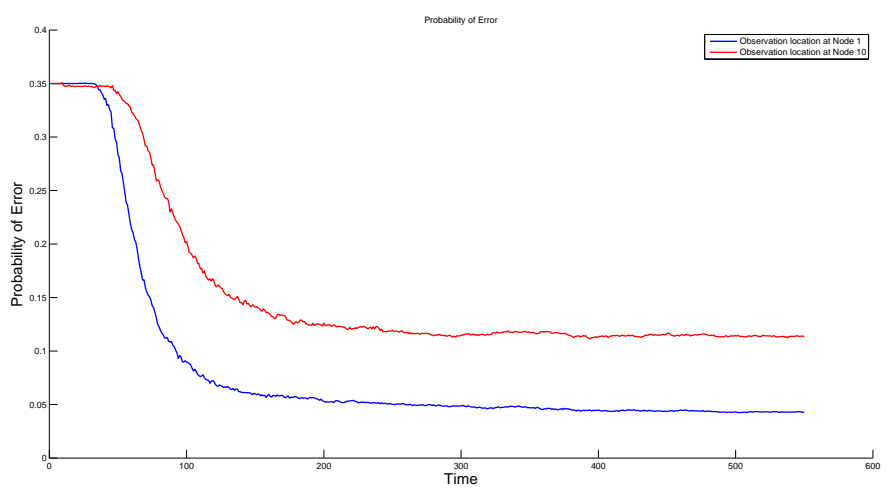

Figure 5: Probability of error versus measurement horizon, for two different observation locations.

node network. From Fig. 8 we see that the bound captures the exponential decreases in the error probability with diminishing measurement noise levels.

\section{Conclusions and Future Work}

We have characterized MAP detection of a link failure in a network synchronization process from noisy local measurements. Specifically, algebraic and graph-theoretic characterizations of the detector form and performance have been obtained, both for both known and stochastic initial states. In the case where the initial state is exactly known (the KS-LFD problem), the link failure can be detected arbitrarily well with a sufficient measurement horizon, under broad assumptions on the network's connectivity. Further, the error probability is shown to fall off exponentially in the measurement horizon in these cases. In contrast, for the SS-LFD problem, formal analyses and simulations suggest that perfect detection is often impossible, but the observations can be leveraged under broad conditions to improve on the a priori decision rule. While our formal analysis shows 


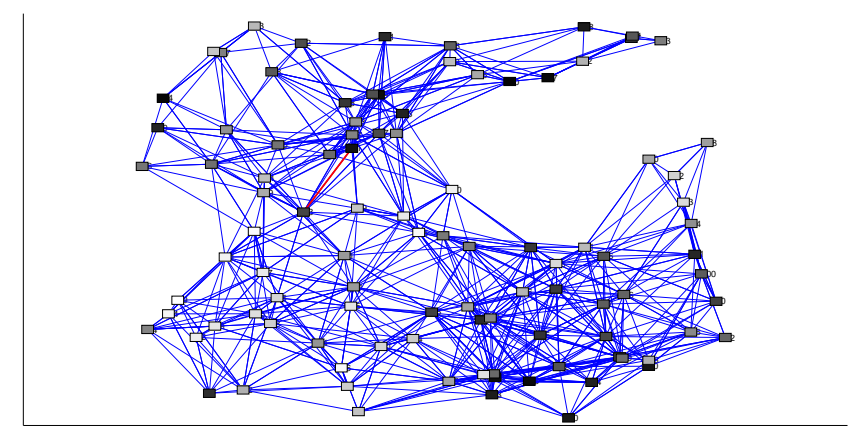

Figure 6: The time required for the probability of error to reach 1 percentage point of steady-state is shown. Darker colors indicate faster detection.

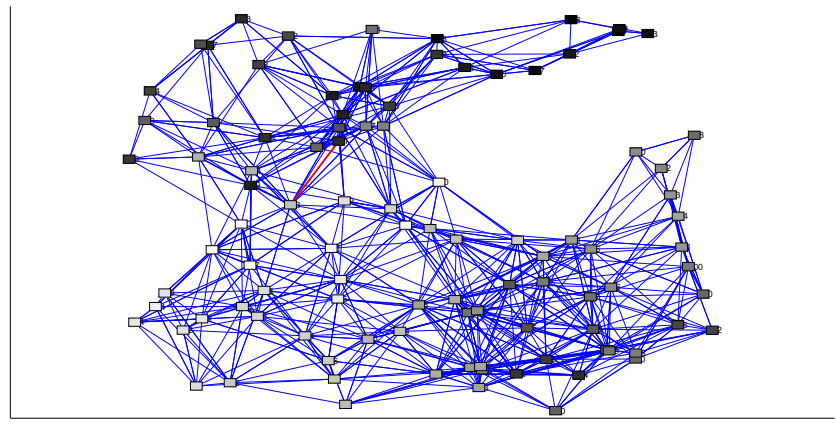

Figure 7: The steady-state probability of error is shown for each measurement location. Darker colors indicate lower error probability.

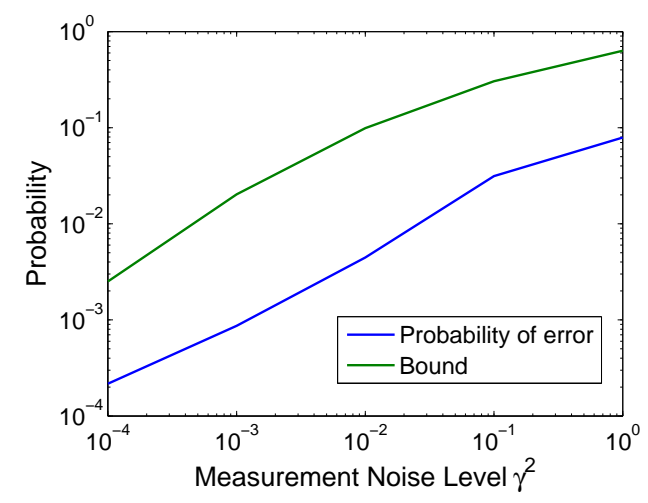

Figure 8: Probability of error and bound as functions of measurement noise level $\gamma^{2}$ 
that the asymptotic rate of the decrease in the error probability (in the known-initial-state case) is the same for all measurement locations, numerical simulations suggest that monitors near the link-failure location exhibit a stronger transient signature and so achieve low-error detection more rapidly. We plan to further investigate this relationship using graph-theoretic methods. We remark that the graph-theoretic characterizations pursued here can be extended towards detection of other forms of structural changes, e.g. node failures, multiple simultaneous link failures or combinations of nodes and link failures.

As future work, we also plan to pursue test-bed implementation of the detection scheme, for a system of realistic size. We envision, particularly, pursuing application in monitoring faults in the electric power grid[25] and in determining communication

failures in WSNs. Several challenges arise in realizing this goal, e.g. related to the volatility of the topology and additional complexity of the synchronization process model.

\section{Appendix A. Proof for the Expansion (11)}

The expansion can be proved using mathmatical induction on $k$. For $k=2$, one can easily see that

$$
\left(A_{i}^{2}-A_{j}^{2}\right)=\left[A_{i}\left(A_{i}-A_{j}\right)+\left(A_{i}-A_{j}\right) A_{j}\right]=\sum_{p=1}^{2} A_{i}^{2-p}\left(A_{i}-A_{j}\right) A_{j}^{p-1}
$$

Let us assume the relationship for $k=n$ :

$$
\left(A_{i}^{n}-A_{j}{ }^{n}\right)=\sum_{p=1}^{n} A_{i}^{n-p}\left(A_{i}-A_{j}\right) A_{j}{ }^{p-1}
$$

Then for $k=n+1$, we have:

$$
\begin{aligned}
\left(A_{i}^{n+1}-A_{j}^{n+1}\right) & =\left(A_{i}\left(A_{i}^{n}-A_{j}^{n}\right)+\left(A_{i}-A_{j}\right) A_{j}^{n}\right) \\
& =A_{i} \sum_{p=1}^{n} A_{i}^{n-p}\left(A_{i}-A_{j}\right) A_{j}{ }^{p-1}+\left(A_{i}-A_{j}\right) A_{j}^{n} \\
& =\sum_{p=1}^{n+1} A_{i}^{n-p}\left(A_{i}-A_{j}\right) A_{j}{ }^{p-1}
\end{aligned}
$$

From this we recover the expansion in (11), with the multiplication of term $e_{s}^{\prime}$.

\section{Appendix B. Theorem Proofs}

Proof of Theorem 1. The result can be proved by analyzing the $k^{\text {th }}$ entry of the differential signature of hypothesis $H_{i}$ over $H_{j}:\left(\mu_{i}-\mu_{j}\right)[k]$. Recognizing that $\mu_{i}-\mu_{j}=$ $\left(O_{i}(T)-O_{j}(T)\right) x[0]$ and using Equation 11, we find that:

$$
\left(\mu_{i}-\mu_{j}\right)[k]=\left\{\begin{array}{r}
0 \\
\text { if } k=0 \\
\sum_{p=1}^{k} e_{s}^{\prime} A_{i}^{k-p}\left(A_{i}-A_{j}\right) A_{j}^{p-1} x[0] \ldots \\
\ldots \text { if } k=1,2, \ldots, T-1
\end{array}\right.
$$


The matrix $A_{i}-A_{j}(i, j \neq 0)$ can be re-written as

$$
A_{i}-A_{j}=w_{P_{j}, Q_{j}} e_{Q_{j}}\left(e_{P_{j}}-e_{Q_{j}}\right)^{\prime}-w_{P_{i}, Q_{i}} e_{Q_{i}}\left(e_{P_{i}}-e_{Q_{i}}\right)^{\prime}
$$

Substituting this in Equation B.1 we immediately see that $\mu_{i}-\mu_{j}$ is the difference of the convolution of the signals in Equation 12. Thus, $\left\|\mu_{i}-\mu_{j}\right\|_{2}^{2}=\sum_{k=0}^{T}\left[\left(r_{(i, j)} * z_{(j, j)}\right)[k]-\left(r_{(i, i)} * z_{(i, j)}\right)[k]\right]^{2}$. If either one of hypotheses is the null hypothesis $(j=0)$, then

$$
A_{0}-A_{i}=w_{P_{i}, Q_{i}} e_{Q_{i}}\left(e_{P_{i}}-e_{Q_{i}}\right)^{\prime}
$$

from which we recover the theorem results.

Proof of THEOREM 2. The statements in the theorem can be proved from the fact that undetectability of the link-failures from the observations $Y_{T}$ requires that $e_{s}^{\prime}\left(A_{i}^{k}-A_{j}^{k}\right)=0$ for every distinct pair of $H_{i}$ and $H_{j}$ and for each $k=1, \cdots, T-1$ (we have excluded the $k=0$ term since it is always zero). In this case, we always decide on the hypothesis that has a higher a priori probability, and hence the link failures are undetectable. Additionally, if $e_{s}^{\prime}\left(A_{i}^{k}-A_{j}^{k}\right)$ is non-zero for some $k$ and some hypotheses $H_{i}$ and $H_{j}$, for a set initial states of non-zero measure, the detector has a non-zero probability of choosing either hypothesis. In consequence, the probability of error is necessarily smaller than the a priori error probability (see [28]). The individual statements can be proved as follows: Statement 1

For a given $k$, if $e_{s}^{\prime}\left(A_{i}^{k}-A_{j}^{k}\right)=0$ for all distinct pairs of hypothesis $H_{i}$ and $H_{j}$, then it is straightforward that $e_{s}^{\prime}\left(A_{0}^{k}-A_{i}^{k}\right)=0$ for all hypotheses $H_{i}$. Similarly if $e_{s}^{\prime}\left(A_{0}^{k}-A_{i}^{k}\right)=$ 0 , we see that $e_{j}^{\prime}\left(A_{i}^{k}-A_{j}^{k}\right)=e_{j}^{\prime} A_{0}^{k}-e_{j}^{\prime} A_{0}^{k}=0$. Thus we only need to show that if $e_{s}^{\prime}\left(A_{0}^{k}-A_{i}^{k}\right)=0$ for $k=1, \cdots, T-1$ and all hypothesis $H_{i}$, for undetectability. The expansion of $e_{s}^{\prime}\left(A_{0}^{k}-A_{i}^{k}\right)$ from Equation 11 immediately proves the result in one direction: if the columns of $\left(A_{0}-A_{i}\right)$ are in the null space of $O_{0}(T)$ for all hypothesis $H_{i}$, then it follows immediately that $e_{s}^{\prime}\left(A_{0}^{k}-A_{i}^{k}\right)=0$ for all $k=1, \cdots, T-1$ for all hypothesis $H_{i}$.

To prove the result in the other direction, we again rely on the expansion of $e_{s}^{\prime}\left(A_{0}^{k}-A_{i}^{k}\right)$ and use an inductive argument. First, from the assumption that $e_{s}^{\prime}\left(A_{0}-A_{i}\right)=0$ (the $k=1$ case), it follows immediately that the columns of $A_{0}-A_{i}$ are in the null space of $e_{s}^{\prime}$.

Inductively, assuming that the columns of $\left(A_{0}-A_{i}\right)$ are in the null space of $e_{s}^{\prime}, e_{s}^{\prime} A_{0}, \cdots, e_{s}^{\prime} A_{0}^{k-2}$ and using the assumption that $e_{s}^{\prime}\left(A_{0}^{k}-A_{i}^{k}\right)=0$, we obtain $e_{s}^{\prime} A_{0}^{k-1}\left(A-A_{i}\right)+\sum_{p=2}^{k} e_{s}^{\prime} A_{0}^{k-p}\left(A_{0}-\right.$ $\left.A_{i}\right) A_{i}^{p-1}=0$ which implies $e_{s}^{\prime} A_{0}^{k-1}\left(A_{0}-A_{i}\right)=0$. This proves that the columns of $\left(A_{0}-A_{i}\right)$ are in the null space of $e_{s}^{\prime} A_{0}^{k-1}$. From the induction, we thus verify that the columns of $\left(A_{0}-A_{i}\right)$ are in the null space of $O_{0}(T)$.

Therefore, we see that if columns of $A_{0}-A_{i}$ are in the null-space of $O_{0}(T)$, the critical link failures are undetectable from $Y_{T}$, regardless of the initial state $x[0]$. Statement 2 From the spectral decomposition of the non-defective matrix $A_{0}$, the $p^{\text {th }}$ entry of the row vector $e_{s}^{\prime} A_{0}^{k}\left(A_{0}-A_{i}\right)$ can be written as:

$$
\begin{cases}w_{P_{i}, Q_{i}} \sum_{l=1}^{n} \lambda_{l(0)}^{k} e_{s}^{\prime} v_{l(0)} w_{l(0)}^{\prime} e_{Q_{i}} & \text { if } p=P_{i} \\ -w_{P_{i}, Q_{i}} \sum_{l=1}^{n} \lambda_{l(0)}^{k} e_{s}^{\prime} v_{l(0)} w_{l(0)}^{\prime} e_{Q_{i}} & \text { if } p=Q_{i} \\ 0 & \text { otherwise }\end{cases}
$$

where $w_{l(0)}^{\prime}$ and $v_{l(0)}$ are the left-and right- eigenvectors corresponding to the eigenvalue $\lambda_{l(0)}, l=1, \ldots, n$, of $A_{0}$. From this decomposition, we obtain the following: if $w_{l(0)}^{\prime} e_{Q_{i}}=0$ 
for every eigenvalue $\lambda_{l(0)}$ for which $e_{s}^{\prime} v_{l(0)} \neq 0$ (i.e. for every observable eigenvalue), then $e_{s}^{\prime} A_{0}^{k}\left(A_{0}-A_{i}\right)$ is a zero vector for all $k$. Therefore, if the $Q_{i}{ }^{t h}$ entry of $w_{l(0)}$ is zero for every observable eigenvalue $\lambda_{l(0)}$, then the $i^{\text {th }}$ critical link failure is undetectable regardless of the observation horizon. If the condition holds for every critical link, then the link-failure problem is undetectable regardless of the observation horizon.

Let us now prove the converse, that the performance will improve if the $Q_{i}{ }^{\text {th }}$ entry of at least one left eigenvector associated with an observable eigenvalue is non-zero. To do so, it is sufficient to prove that $\mu_{0}$ and $\mu_{i}$ are different: in this case, the decision rule differentiates between the two hypotheses, and hence the probability of error in detection improves.

Let us verify that $\mu_{0}$ and $\mu_{i}$ are different by considering the energy of the differential signature of $H_{i}$ over the null hypothesis $H_{0}:\left\|\mu_{0}-\mu_{i}\right\|_{2}^{2}$. We note that this difference is the energy of the differential signature which is defined as the convolution of the signals:

- $r_{(i, i)}[k]=\sum_{l=1}^{n} \lambda_{l(i)}^{k}\left(e_{P_{i}}-e_{Q_{i}}\right)^{\prime} v_{l(i)} w_{l(i)}^{\prime} x[0]$ and

- $z_{(0, i)}[k]=w_{P_{i}, Q_{i}} \sum_{l=1}^{n} \lambda_{l(0)}^{k} e_{s}^{\prime} v_{l(0)} w_{l(0)}^{\prime} e_{Q_{i}}$,

where $w_{l(i)}^{\prime}$ and $w_{l(i)}$ are the left- and right- eigenvectors corresponding to the eigenvalue $\lambda_{l(i)}, l=1, \ldots, n$, of $A_{i}$ for $i=1, \cdots, m$.

Thus, if both signals are non-zero then $\left\|\mu_{0}-\mu_{i}\right\|_{2}^{2}$ has to be non-zero. It is clear that if the $Q_{i}{ }^{\text {th }}$ entry of at least one left-eigenvector associated with an observable eigenvalue is non-zero, then $z[k]$ is non-zero. Under generic initial states $w_{l(i)}^{\prime} x[0] \neq 0$ for at least one eigenvalue of $A_{i}$. Notice that only a special set of initial states $x[0]$ will make $w_{l(i)}^{\prime} x[0]=0$ for every nonzero eigenvalue of $A_{i}$. Furthermore since the right eigenvectors span $\mathfrak{R}^{n}$, we know that there is at least one right eigenvector $v_{l(i)}$ such that the $P_{i}^{\text {th }}$ and $Q_{i}{ }^{\text {th }}$ entries are different. Therefore the differential signature of the failure is non-zero and hence the performance of the detector improves. Statement 3

Consider the critical link $l_{i}$. Notice that any change edge weight, $w_{P_{i}, Q_{i}}$, is reflected in both $A_{0}\left(Q_{i}, P_{i}\right)$ and $A_{0}\left(Q_{i}, Q_{i}\right)$. The derivative of an eigenvalue $\lambda_{l(0)}$ of $A_{0}$ with respect to $w_{P_{i}, Q_{i}}$ is given by:

$$
\frac{\partial\left(\lambda_{l}\right)}{\partial w_{P_{i}, Q_{i}}}=w_{l(0)}\left(Q_{i}\right)\left(v_{l(0)}\left(P_{i}\right)-v_{l(0)}\left(Q_{i}\right)\right)
$$

where $w_{l(0)}\left(P_{i}\right)$ and $v_{l(0)}\left(P_{i}\right)$ are the $P_{i}^{\text {th }}$ entry of the left- and right-eigenvectors of the eigenvalue $\lambda_{l(0)}$ of the nominal state matrix $A_{0}$. We first note that the dominant eigenvalue of $A_{0}$ is insensitive to the link failure and is always equal to unity. From Equation B.3, we also note that if $w_{l(0)}\left(Q_{i}\right)=0$ then eigenvalue $\lambda_{l}$ is insensitive to the failure of critical link $l_{i}$. From Statement 2, we see that if $w_{l(0)}\left(Q_{i}\right)=0$ for all the observable eigenvalues, i.e. the failure of $l_{i}$ is undetectable regardless of the initial state, then observable eigenvalues are insensitive to failures of $l_{i}$. If the same is true for all critical links $l_{i}$, then the MAP detector is unable to detect any link failure regardless of the initial state.

Proof of Theorem 3. To prove the graph-theoretic result for undetectability, we consider each hypothesis $H_{i}$ individually. Specifically for hypothesis $H_{i}$, we show that if 
there are no directed paths from $Q_{i}$ to the observation location (component $s$ ), then $e_{s}^{\prime} A_{0}^{k}\left(A_{0}-A_{i}\right)=0$. Proving this for every hypothesis, we arrive at the result presented in the theorem using the matrix-theoretic condition for undetectability from Theorem 2. Let us begin our analysis by defining a new graph.

Specifically, let us define a graph $\widehat{\Gamma}=(\mathcal{V}, \widehat{E}: \widehat{W})$, whose adjacency matrix is $A_{0}$. That is, the graph $\widehat{\Gamma}$ is defined to have an edge $\left(i, j: w_{i j}\right)$ if and only if $A_{0}(j, i) \neq 0$. We stress that each vertex of $\widehat{\Gamma}$ has a self-loop (an edge back to itself). Thus, if there is a directed walk from vertex $i$ to vertex $j$ whose length is $k$ in $\widehat{\Gamma}$ then there is also a directed walk of any length greater than $k$ from $i$ to $j$ in $\widehat{\Gamma}$.

We recall that $A_{0}=I-L$, where $L$ is the Laplacian matrix associated with the graph $\Gamma$. Thus, we can relate the graph $\widehat{\Gamma}$ to $\Gamma$ as follows: there are directed walks of any length greater than or equal to $k$ (and none of length less than $k$ ) from vertex $i$ to vertex $j$ in $\widehat{\Gamma}$, if and only if there is a directed path of length $k$ in $\Gamma$.

Let us now provide a graph-theoretic characterization of the condition for link-failure undetectability. That is, we are interested in providing a graph-theoretic characterization of the condition: $e_{s}^{\prime} A_{0}^{k}\left(A_{0}-A_{i}\right)=0$ for all $k=0, \cdots, T-2$.

Specifically, the $p^{\text {th }}$ entry of the row vector $e_{s}^{\prime} A_{0}^{k}\left(A_{0}-A_{i}\right)$ can be written as:

$$
\begin{cases}\alpha \sum_{k \text {-walks }} \mathcal{W}_{\widehat{\Gamma}}\left(Q_{i}, s\right) & \text { if } p=P_{i} \\ -\beta \sum_{k \text {-walks }} \mathcal{W}_{\widehat{\Gamma}}\left(Q_{i}, s\right) & \text { if } p=Q_{i} \\ 0 & \text { otherwise }\end{cases}
$$

where $\alpha$ and $\beta$ are some positive constants, and $\mathcal{W}_{\widehat{\Gamma}}\left(Q_{i}, s\right)$ is the weight of a directed walk from vertex $Q_{i}$ to vertex $s$ in the graph $\widehat{\Gamma}$.

Given that the weight of any directed walk in $\widehat{\Gamma}$ is positive, $e_{s}^{\prime} A_{0}^{k}\left(A_{0}-A_{i}\right)$ equals 0 for $k=0, \cdots, T-2$ if and only if there is no directed walk from vertex $Q_{i}$ to vertex $s$ of length $k$. Moreover, if there is no such directed walk sufficiently long, i.e. length greater or equal to $n-1$, there is no such directed walk at all. From the equivalence between walks in $\widehat{\Gamma}$ and paths in $\Gamma$, link failures are thus undetectable regardless of the initial state and observation horizon if and only if there are no directed paths from vertex $Q_{i}$ to the observation vertex, s. Combining the result for all hypotheses leads us to the graph-theoretical condition for undetectability in the theorem statement.

The smallest observation horizon $[0, T]$ after which the MAP detector improves on the a priori probability is given by the smallest $k$ such that $e_{s}^{\prime} A_{0}^{k}\left(A_{0}-A_{i}\right) \neq 0$ for some $H_{i}$. This is equivalent to the smallest $k$ such that a directed path of length $k$ exists from $Q_{i}$ to s, i.e. $\sum_{k \text {-walks }} \mathcal{W}_{\widehat{\Gamma}}\left(Q_{i}, s\right) \neq 0$.

Let us now show that the existence of paths from $Q_{i}$ to $s$ improves the probability of error. This part of the proof relies on the differential signature of $H_{i}$ over the nominal $H_{0}$. We can obtain a graph-theoretical characterization on the signals $r_{(i, i)}[k]$ and $z_{(0, i)}[k]$ (Theorem 1). Specifically, considering the graph $\widehat{\Gamma}$ we see that

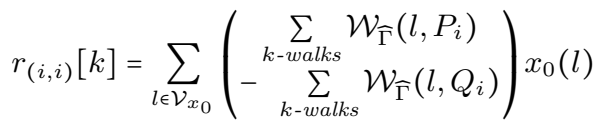

$$
\begin{aligned}
& z_{(0, i)}[k]=w_{P_{i} Q_{i}} \sum_{k \text {-walks }} \mathcal{W}_{\widehat{\Gamma}}\left(Q_{i}, s\right)
\end{aligned}
$$


where $s$ is the observation location, $\mathcal{V}_{x_{o}}$ contains the vertices whose corresponding initial state components are non-zero, $x_{0}(l)$ represents the initial state at vertex $l$, and $\mathcal{W}_{\widehat{\Gamma}}(i, j)$ denotes the weight of a directed walk from vertex $i$ to vertex $j$ in the graph $\widehat{\Gamma}$.

The line failure $l_{i}$ is detectable if and only if the failure signature is nonzero for at least one time on the time interval $[0, T]$, which is achieved if and only if the signals $r_{(i, i)}[k]$ and $z_{(0, i)}[k]$ are nonzero for some time $k$. The space of initial states for which $\sum_{l \in \mathcal{V}_{x_{0}}} \sum_{k \text {-walks }} \mathcal{W}_{\widehat{\Gamma}}\left(l, Q_{i}\right) x_{0}(l)$ equals $\sum_{l \in \mathcal{V}_{x_{0}}} \sum_{k \text {-walks }} \mathcal{W}_{\widehat{\Gamma}}\left(l, P_{i}\right) x_{0}(l)$ has dimension less than $n$. Hence, the two expressions are unequal for a generic initial state, and $r_{(i, i)}[k]$ is necessarily non-zero.

Thus, to prove the theorem, it is enough to show that $z_{(0, i)}[k] \neq 0$ for some $k$ if and only if there is exist a directed path in $\Gamma$ from $Q_{i}$ to the observation location. This is verified directly by Equation B.5, since $z_{(0, i)}[k] \neq 0$ if and only if there is at least one directed walk of length $k$ from vertex $Q_{i}$ to vertex $s$.

Proof of Theorem 4. In order to prove the first part of the theorem, let us examine the 2-norm of the vector $\mu_{i}-\mu_{j}$ (energy of the differential signature of $H_{i}$ over $H_{j}$ ) by studying its entries. Specifically, the $k^{\text {th }}$ entry $(k \in\{0, \cdots, T-1\})$ is $e_{s}^{\prime}\left(A_{i}^{k}-A_{j}^{k}\right) x[0]$. From the spectral properties of $A_{i}$ and $A_{j}$, we see that for a large $k$

$$
\begin{aligned}
e_{s}^{\prime}\left(A_{i}^{k}-A_{j}^{k}\right) x[0] & \rightarrow e_{s}^{\prime}\left(v_{1(i)} w_{1(i)}^{\prime}-v_{1(j)} w_{1(j)}^{\prime}\right) x[0] \\
& =\left(w_{1(i)}^{\prime}-w_{1(j)}^{\prime}\right) x[0]
\end{aligned}
$$

We observe that the entries of $\mu_{i}-\mu_{j}$ approach non-zero constants if and only if $w_{1(i)}^{\prime} x[0] \neq$ $w_{1(j)}^{\prime} x[0]$. In this case, as the observation horizon becomes sufficiently large $(T \rightarrow \infty)$, $\left\|\mu_{i}-\mu_{j}\right\|_{2}$ approaches $+\infty$. From this, one recovers that $\operatorname{erf}\left(\frac{\left\|\mu_{j}-\mu_{i}\right\|_{2}}{2 \gamma}+\frac{\gamma \log \frac{p_{i}}{p_{j}}}{\left\|\mu_{j}-\mu_{i}\right\|_{2}}\right) \rightarrow 1$ as $T \rightarrow \infty$. If this condition holds for every pair of hypotheses $H_{i}$ and $H_{j}$, we see that $P_{\text {err }} \rightarrow 0$ as $T \rightarrow \infty$.

Next, we show that for generic initial state $x[0]$, strong connectivity of the nominal network graph (hypothesis $H_{0}$ ) is sufficient for asymptotic perfect detection. To prove this, we use the eigenvector majorization approach introduced in [32]. For every hypothesis $H_{i}, i \geq 1$, we show that $w_{1(0)} \neq w_{1(i)}$. From this we argue that $w_{1(i)}^{\prime} x[0] \neq w_{1(j)}^{\prime} x[0]$ for every pair of hypotheses $H_{i}$ and $H_{j}$. Let us begin our analysis by comparing $w_{1(i)}$ to $w_{1(0)}$ for some $H_{i}$

Without loss of generality consider that the critical link for $H_{i}$ is from the component 2 to $1^{3}$ and hence $A_{i}=A_{0}-w_{21} e_{1}\left(e_{2}-e_{1}\right)^{\prime}$. The first column of $A_{i}$ is $w_{21} e_{1}$ and the second column is $-w_{21} e_{1}$. If $\Gamma$ is strongly connected, the increment/decrement of the entries $A_{0}(1,1)$ and $A_{0}(1,2)$ of $A_{0}$ do not change the dominant eigenvalue $\lambda=1$ per our definition of $A_{i}$ and also maintain the positivity of the left eigenvector. Hence, we can rewrite the dominant left eigenvector (for some normalization) as $\tilde{w}_{1(i)}^{\prime}=\left[\begin{array}{lll}w_{1} & w_{2: n}^{\prime}+\mathbf{q}_{\mathbf{i}}{ }^{\prime}\end{array}\right]$, where $w_{2: n}^{\prime}=\left[\begin{array}{lll}w_{2} & \cdots & w_{n}\end{array}\right]$ contains the last $n-1$ components of the left-eigenvector of $A_{0}$ and $\mathbf{q}_{\mathbf{i}}{ }^{\prime}$ is a $n-1$ vector. Notice that we have used $\tilde{w}_{1(i)}$ to denote this specific renormalization. We shall prove that $\mathbf{q}_{\mathbf{i}}$ is component-wise non-positive.

\footnotetext{
${ }^{3}$ As per our notations, the critical link for $H_{i}$ is between components $P_{i}$ and $Q_{i}$. For the purposes for the proof we temporarily assume that $P_{i}$ and $Q_{i}$ have been relabeled as 2 and 1 respectively.
} 
In order to prove this condition, we work with the eigenvector equation $w_{1(i)}^{\prime} A_{i}=w_{1(i)}^{\prime}$, and consider the last $n-1$ equations in the system. Substituting $w_{1(i)}$ and using the eigenvector equation $\tilde{w}_{1,(i)}^{\prime} A_{i}=\tilde{w}_{1,(i)}^{\prime}$, we find that

$$
\mathbf{q}_{\mathbf{i}}{ }^{\prime}\left(I-A_{0}(2: n, 2: n)\right)=\left[\begin{array}{llll}
-\alpha & 0 & \cdots & 0
\end{array}\right]
$$

where $A_{0}(2: n, 2: n)$ is the submatrix of $A_{0}$ formed by the rows and columns 2 to $n$ and $\alpha$ is a strictly positive quantity. From classical results, we have that dominant eigenvalue of $A_{0}(2: n, 2: n)$ is less than 1 . Thus, $I-A_{0}(2: n, 2: n)$ is a nonsingular M-matrix [33] and hence its inverse is element-wise nonnegative. In fact, $\left(I-A_{0}(2: n, 2: n)\right)^{-1}$ has at least one strictly positive entry on each row/column, so $\mathbf{q}_{\mathbf{i}}{ }^{\prime} \leq 0$ has at least one nonzero entry. Hence, when $\tilde{w}_{1(i)}$ is renormalized to $w_{1(i)}$, we see that $w_{1(i)}(1) \geq w_{1(0)}(1)$ where $w_{1(i)}(1)$ is the first entry of $w_{1(i)}$. In particular, the $Q_{i}^{\text {th }}$ term increases for every hypothesis $H_{i}$. Therefore, if $H_{i}$ and $H_{j}$ are such that $Q_{i} \neq Q_{j}$ (critical links do not end at a common vertex), $w_{1(i)}^{\prime} x[0] \neq w_{1(j)}^{\prime} x[0]$ for generic $x[0]$. We are left to show that this condition holds for two hypotheses $H_{i}$ and $H_{j}$ where the critical links end at a common vertex.

Again, without loss of generality we assume that the critical link for hypothesis $H_{i}$ is from the component 2 to 1 and that for the hypothesis $H_{j}$ is the from the component 3 to 1. Subtracting Equation B.7 for $H_{j}$ from that of $H_{i}$

$$
\left(\mathbf{q}_{\mathbf{i}}-\mathbf{q}_{\mathbf{j}}\right)^{\prime}\left(I-A_{0}(2: n, 2: n)\right)=\left[\begin{array}{llll}
-\alpha & \beta & \cdots & 0
\end{array}\right]
$$

where $\alpha$ and $\beta$ are strictly positive quantities. Since, $\left(I-A_{0}(2: n, 2: n)\right)$ is a non-singular $M$-matrix, we can see that $\mathbf{q}_{\mathbf{i}} \neq \mathbf{q}_{\mathbf{j}}$. Therefore, $w_{1(i)}(1)$ and $w_{1(j)}(1)$ increase by different amounts. Thus for generic initial state $x[0]$, we can conclude that the strongly connected network graph for the nominal dynamics is sufficient for asymptotic perfect detection.

Proof of TheORem 5. The exponential decay in the error probability follows from the derived algebraic bounds for $P_{\text {err }}$. Specifically, let $P_{\text {err }}(k)$ be the detection error probability for a measurement horizon $[0, k]$. Also, we let $P_{\text {err }}^{*}=\sup P_{\text {err }}(k)<1$ be the supremum of the probability of error over time, and note that $P_{\text {err }}^{*}{ }^{k}$ is upper-bounded by the a priori error probability. From Equation B.6, it is easy to see that, for large $k,\left\|\mu_{i}-\mu_{j}\right\|_{2}$ is within $\mathcal{O}(1)$ of $e_{s}^{\prime} \mathbf{1}\left(w_{1(i)}^{\prime}-w_{1(j)}^{\prime}\right) x[0] \sqrt{k}$ (i.e., near this value, to within a constant deviation). For notational convenience, let us define $\alpha_{(i, j)}=\left(w_{1(i)}^{\prime}-w_{1(j)}^{\prime}\right) x[0]$. Let $\alpha_{1}=\min _{i, j} \alpha_{(i, j)}$. From Equation 10, we see that, for large $k$, the probability of error is upper bounded by

$$
\begin{aligned}
P_{e r r}(k) & \leq \frac{m}{2}-\frac{1}{2} \sum_{i=0}^{m} p_{i} \sum_{j \neq i} \operatorname{erf}\left(\alpha_{(i, j)} \sqrt{k}\right) \\
& \leq \frac{m}{2}\left[1-\operatorname{erf}\left(\alpha_{1} \sqrt{k}\right)\right]
\end{aligned}
$$

which can be upper bounded by $\exp \left(-\alpha_{1}^{2} k\right)$ using the Chernoff inequality (see [34]).

From this exponential bound for $P_{\text {err }}$ for large $k$ together with the fact that $P_{\text {err }}^{*}$ is upper bounded, the theorem statement follows immediately (for $\eta<\exp \left(-\alpha_{1}^{2}\right)$ and sufficiently-large $\alpha$ ). 
ProOF OF THEOREM 6. The condition for undetectability in the theorem statement comes directly from Equation 14. Specifically, if $\Sigma_{i}=\Sigma_{j}$, for every pair of hypotheses $H_{i}$ and $H_{j}$, then the hypothesis test reduces to comparing the a priori error probabilities, and so the detector's performance is no better than the a priori. It is also straightforward to see that $\Sigma_{i}=\Sigma_{j}$, for every pair of hypothesis $H_{i}$ and $H_{j}$ if and only $\Sigma_{0}=\Sigma_{i}$ for every hypothesis $H_{i}(i \geq 1)$.

If $\Sigma_{0} \neq \Sigma_{i}$ for some hypothesis $H_{i}$, there is a set of observation sequences of nonzero probability, such that the detector's choice is different from the a priori most likely hypothesis. It is easy to check, in turn, that the probability of error improves upon the a priori.

Proof of TheOrem 7. For every hypothesis $H_{i}$, if the columns of $\left(A_{0}-A_{i}\right)$ are in the null-space of $O_{0}(T)$ then $\Sigma_{0}=\Sigma_{i}$ for all $i$, i.e. then the link-failure is undetectable for the random-initial-state case. Notice that this condition is exactly the same for the known-initial-state case (Statement 1 of Theorem 2). The equivalence of Theorem 3 and Theorem 2 proves one direction of the theorem statement.

This condition is also the matrix-theoretic condition for undetectability of critical link failures in the known $x[0]$ case. In Theorem 3 we showed that this condition is satisfied if and only if there are no directed paths to the observation location from any of the trailing ends $Q_{i}$. Hence the link failures are undetectable regardless the observation horizon, $[0, T]$ if there are no directed paths from the trailing ends of any of the critical links to any of the observation locations.

Thus, we are left to prove the reverse direction, i.e. if there is a directed path to the observation location $s$ from some $Q_{i}$ for $i=1, \ldots, m$, then $\Sigma_{0} \neq \Sigma_{i}$. To prove this, we construct the graphs $\widehat{\Gamma}$ and $\widehat{\Gamma_{i}}$ with self loops by considering $A_{0}$ and $A_{i}$ as adjacency matrices (as we did in the proof for Theorem 3), and examine the entry at the $p^{\text {th }}$ row and $q^{\text {th }}$ column $(p \leq q W L O G)$ of $\Sigma_{0}-\Sigma_{i}$. Specifically, we can see that

$$
\begin{aligned}
& \left(\Sigma_{0}-\Sigma_{i}\right)_{p, q}=C A_{0}^{p} A_{0}^{\prime q} C^{\prime}-C A_{i}^{p} A_{i}^{\prime q} C^{\prime} \\
& =\sum_{l=1}^{n}\left(\begin{array}{c}
\mathcal{T} \mathcal{W}_{\widehat{\Gamma}}(l, s, p) \mathcal{T} \mathcal{W}_{\widehat{\Gamma}}(l, s, q) \\
-\mathcal{T} \mathcal{W}_{\widehat{\Gamma}_{i}}(l, s, p) \mathcal{T} \mathcal{W}_{\widehat{\Gamma}_{i}}(l, s, q)
\end{array}\right)
\end{aligned}
$$

where for clarity we have employed the notation

$$
\mathcal{T} \mathcal{W}_{\widehat{\Gamma}}(i, s, p)=\sum_{p-w a l k s} \mathcal{W}_{\widehat{\Gamma}}(i, s)
$$

to represent the total weight of all the p-length directed walks in $\widehat{\Gamma}$ from $i$ to $s$, or equivalently the total weight of all the p-length directed paths in $\Gamma$ from $i$ to $s$.

If the critical link $l_{i}$ was part of a directed path of length $p$ in $\Gamma$ from some vertex $l \in \mathcal{V}$ to observation location $s$, then $\Gamma_{i}$ would certainly have fewer p-length paths from $l$ to $s$. We recall that $p$-length paths in $\Gamma$ and $\Gamma_{i}$ are also p-length paths in $\widehat{\Gamma}$ and $\widehat{\Gamma_{i}}$. Therefore, it follows that if $l_{i}$ was part of a directed path of length $p$ in $\Gamma$ from $l$ to $s$, then $\mathcal{T W}_{\widehat{\Gamma}}(l, s, p)>\mathcal{T} \mathcal{W}_{\widehat{\Gamma}_{i}}(l, s, p)^{4}$. Therefore, $\Sigma_{0} \neq \Sigma_{i}$.

\footnotetext{
${ }^{4}$ Since $q \geq p$ number of $q$-length paths are always greater than (or equal to) the number of $p$-length paths in both $\Gamma$ and $\Gamma_{i}$.
} 
Further, $l_{i}$ can be part of a directed path from $l$ to $s$ if and only if there is a directed path from $Q_{i}$ to $s$, which is the trailing end of $l_{i}$. Therefore, we conclude that if there is a directed path to observation location from $Q_{i}$, then $\Sigma \neq \Sigma_{i}$. Combining this conclusion for every hypothesis $H_{i}$, we arrive at the theorem statement.

Proof of Theorem 8. To develop the bounds, let us denote by $\tilde{H}$ the final hypothesis chosen by the MAP detector based on the observations $Y_{T}$. Notice that the probability of error can be expressed as $P_{\text {err }}=\sum_{i=0}^{m} p_{i} \sum_{j \neq i} \operatorname{Pr}\left(\tilde{H}=H_{j} \mid H_{i}\right)$.

For some hypothesis $H_{j} \neq H_{i}$,

$$
\operatorname{Pr}\left(\tilde{H}=H_{j} \mid H_{i}\right)=\operatorname{Pr}\left(Y_{T}^{\prime}\left(\Sigma_{i}^{-1}-\Sigma_{j}^{-1}\right) Y_{T} \leq \zeta_{i, j} \mid H_{i}\right)
$$

where $\left.\zeta_{i, j}=2 \log \left(\frac{p_{j}}{p_{i}}\right)+\log \left|\Sigma_{i} \Sigma_{j}^{-1}\right|\right)$. We note that $\zeta_{i j}$ is anti-symmetric with respect to the indices, i.e. $\zeta_{i, j}=-\zeta_{j, i}$.

Quadratic forms involving multi-variate Gaussian random variables do not readily yield a closed form expressions for their density/distribution functions. However, closed form expressions can be found for their moment generating functions (see [35]). Specifically,

$$
\begin{aligned}
\theta_{i, j}(\bar{s}) & \left.=E\left[e^{\bar{s} Y_{T}^{\prime}\left(\Sigma_{i}^{-1}-\Sigma_{j}^{-1}\right) Y_{T}}\right) \mid H_{i}\right] \\
& =\left|I-2 \bar{s}\left(I-\Sigma_{j}^{-1} \Sigma_{i}\right)\right|^{-\frac{1}{2}}
\end{aligned}
$$

where we use $\theta_{i, j}(\bar{s})$ to denote the moment generating function of $Y_{T}^{\prime}\left(\Sigma_{i}^{-1}-\Sigma_{j}^{-1}\right) Y_{T}$ conditioned on $H_{i}$.

The explicit expressions (B.11) allow us to use Chernoff's inequality for characterizing $\operatorname{Pr}\left(\tilde{H}=H_{j} \mid H_{i}\right)$. Specifically,

$$
\operatorname{Pr}\left(\tilde{H}=H_{j} \mid H_{i}\right) \leq \min \left[1,\left(\inf _{\bar{s}} e^{\bar{s} \zeta_{i, j}} \theta_{i, j}(-\bar{s})\right)\right]
$$

where, for the lower tail probability, we have used that $\operatorname{Pr}\left(Y_{T}^{\prime}\left(\Sigma_{i}^{-1}-\Sigma_{j}^{-1}\right) Y_{T} \leq \zeta_{i, j} \mid H_{i}\right)=$ $\operatorname{Pr}\left(-Y_{T}^{\prime}\left(\Sigma_{i}^{-1}-\Sigma_{j}^{-1}\right) Y_{T} \geq-\zeta_{i, j} \mid H_{i}\right)$, and also that $\theta_{X}(-\bar{s})=\theta_{-X}(\bar{s})$ for any random variable $X$. We note that we have restricted the right-hand side to be unity at most.

The upper-bound on $P_{\text {err }}$ in the theorem statement follows the above inequality. The $1-\max _{i}\left(p_{i}\right)$ term is included to ensure that the upper-bound for $P_{\text {err }}$ is always upperbounded by the a priori probability of error.

\section{References}

[1] A. Arenas, A. Díaz-Guilera, J. Kurths, Y. Moreno, C. Zhou, Synchronization in complex networks, Physics Reports 469 (3) (2008) 93-153.

[2] L. Xiao, S. Boyd, S.-J. Kim, Distributed average consensus with least-mean-square deviation, Journal of Parallel and Distributed Computing 67 (1) (2007) 33-46.

[3] A. E. Motter, C. Zhou, J. Kurths, Network synchronization, diffusion, and the paradox of heterogeneity, Physical Review E 71 (1) (2005) 016116.

[4] F. Pasqualetti, A. Bicchi, F. Bullo, Consensus computation in unreliable networks: A system theoretic approach, Automatic Control, IEEE Transactions on 57 (1) (2012) 90-104.

[5] D. J. Watts, S. H. Strogatz, Collective dynamics of small-worldnetworks, nature 393 (6684) (1998) 440-442. 
[6] S. H. Strogatz, Exploring complex networks, Nature 410 (6825) (2001) 268-276.

[7] A.-L. Barabási, R. Albert, H. Jeong, Scale-free characteristics of random networks: the topology of the world-wide web, Physica A: Statistical Mechanics and its Applications 281 (1) (2000) 69-77.

[8] S. Roy, A. Saberi, K. Herlugson, A control-theoretic perspective on the design of distributed agreement protocols, International Journal of Robust and Nonlinear Control 17 (10-11) (2007) 10341066.

[9] S. Maslov, K. Sneppen, A. Zaliznyak, Detection of topological patterns in complex networks: correlation profile of the internet, Physica A: Statistical Mechanics and its Applications 333 (2004) 529-540.

[10] P. Barooah, H. Chenji, R. Stoleru, T. Kalmár-Nagy, Cut detection in wireless sensor networks, Parallel and Distributed Systems, IEEE Transactions on 23 (3) (2012) 483-490.

[11] F. Ciulla, N. Perra, A. Baronchelli, A. Vespignani, Damage detection via shortest-path network sampling, Physical Review E 89 (5) (2014) 052816.

[12] L. Lü, T. Zhou, Link prediction in complex networks: A survey, Physica A: Statistical Mechanics and its Applications 390 (6) (2011) 1150-1170.

[13] M. A. Kramer, U. T. Eden, S. S. Cash, E. D. Kolaczyk, Network inference with confidence from multivariate time series, Physical Review E 79 (6) (2009) 061916.

[14] Y. Yuan, G.-B. Stan, S. Warnick, J. Goncalves, Robust dynamical network structure reconstruction, Automatica 47 (6) (2011) 1230-1235.

[15] Y. Wan, S. Roy, M. Xue, V. Katragadda, Estimating modes of a complex dynamical network from impulse response data: Structural and graph-theoretic characterizations, International Journal of Robust and Nonlinear Control.

[16] J. J. Gertler, Survey of model-based failure detection and isolation in complex plants, Control Systems Magazine, IEEE 8 (6) (1988) 3-11.

[17] R. M. Ferrari, T. Parisini, M. M. Polycarpou, Distributed fault detection and isolation of largescale discrete-time nonlinear systems: An adaptive approximation approach, Automatic Control, IEEE Transactions on 57 (2) (2012) 275-290.

[18] R. Dhal, J. Abad Torres, S. Roy, Link-failure detection in network synchronization processes, in: Global Conference on Signal and Information Processing (GlobalSIP), 2013 IEEE, 2013, pp. 779-782. doi:10.1109/GlobalSIP.2013.6737007.

[19] F. R. Chung, Spectral graph theory, Vol. 92, Amer Mathematical Society, 1997.

[20] J. V. Below, Can one hear the shape of a network?, in: Partial differential equations on multistructures (Luminy, 1999), Vol. 219 of Lecture Notes in Pure and Appl. Math., Dekker, New York, 2001, pp. 19-36.

[21] T. Zhou, M. Zhao, C. Zhou, Synchronization on effective networks, New Journal of Physics 12 (4) (2010) 043030.

[22] T. Nishikawa, A. E. Motter, Synchronization is optimal in nondiagonalizable networks, Physical Review E 73 (6) (2006) 65106.

[23] W. Ren, R. W. Beard, Consensus seeking in multiagent systems under dynamically changing interaction topologies, Automatic Control, IEEE Transactions on 50 (5) (2005) 655-661.

[24] D.-U. Hwang, M. Chavez, A. Amann, S. Boccaletti, Synchronization in complex networks with age ordering, Physical review letters 94 (13) (2005) 138701.

[25] J. Valdez, X. Zhang, J. A. Torres, S. Roy, Fast fault location in power transmission networks using transient signatures from sparsely-placed synchrophasors, in: North American Power Symposium (NAPS), 2014, IEEE, 2014, pp. 1-6.

[26] W. J. Rugh, Linear system theory, Prentice-Hall, Inc., 1996.

[27] H. Kwakernaak, R. Sivan, Linear optimal control systems, Vol. 172, Wiley-Interscience New York, 1972 .

[28] R. G. Gallager, Stochastic processes: theory for applications, Cambridge University Press, 2013.

[29] M. Xue, E. Yeung, A. Rai, S. Roy, Y. Wan, S. Warnick, Initial-condition estimation in network synchronization processes: algebraic and graphical characterizations of the estimator, in: AIAA Guidance, Navigation and Control Conference, Portland, OR, 2011, pp. 8-11.

[30] Y.-Y. Liu, J.-J. Slotine, A.-L. Barabási, Observability of complex systems, Proceedings of the National Academy of Sciences 110 (7) (2013) 2460-2465.

[31] E. W. Weisstein, Hyperboloid, MathWorld-A Wolfram Web Resource."http://mathworld.wolfram.com/Hyperboloid.html".

[32] S. Roy, A. Saberi, Y. Wan, Majorizations for the dominant eigenvector of a nonnegative matrix, in: American Control Conference, Westin Seattle Hotel, Seattle, Washington, USA, 2008, pp. 1965-1966. 
[33] A. Berman, R. J. Plemmons, Nonnegative matrices, The Mathematical Sciences, Classics in Applied Mathematics, 9.

[34] H. Stark, J. W. Woods, Probability, random processes, and estimation theory for engineers, Prentice-Hall Englewood Cliffs (NJ), 1986, pp. 202-203.

[35] A. C. Rencher, G. B. Schaalje, Linear models in statistics, Wiley, 2008. 